\title{
Singular perturbation analysis of cAMP signalling in Dictyostelium discoideum aggregates
}

\author{
Gabriela Liţcanu* and Juan J. L. Velázquez** \\ *Institute of Mathematics "O. Mayer", Romanian Academy, \\ Bd. Carol I, 700505 Iasi, Romania. e-mail: gabrielal@academie.is.edu.ro, lorelai@mimuw.edu.pl \\ **Departamento de Matemática Aplicada, Facultad de Matemáticas, \\ Universidad Complutense de Madrid, 28040 Madrid, Spain. e-mail: JJ_Velazquez@mat.ucm.es
}

\begin{abstract}
In this paper, we use singular perturbation methods to study the structure of travelling waves for some reaction-diffusion models obtained from the Martiel-Goldbeter and Goldbeter-Segel's models of cAMP signalling in Dictyostelium discoideum. As a consequence, we derive analytic formulae for quantities like wave speed, maximum concentration and other magnitudes in terms of the different biochemical constants that appear in the model.
\end{abstract}

Mathematics Subject Classification (2000): 35B25, 35K57, 92C37

Key words or phrases: Singular perturbation methods - Travelling waves - Reaction-diffusion systems

\section{Introduction}

This paper is concerned with the analysis of some reaction-diffusion systems modelling the propagation of cAMP signals during the aggregation of the amoeboid microorganism Dictyostelium discoideum ( $D d$ hereafter).

As the life cycle of $D d$ incorporates a number of basic processes that occur throughout developmental biology, this cellular slime mould has been studied for a long time as a model system for biological pattern formation. Taking into account the experimental data for the biological processes that occur in this organism (see, for example, [1], [3], [4], [6], [17]), several studies have analyzed the developmental cycle of $D d$ by means of different mathematical models (see, for instance, [2], [5], [9], [10], [12], [13], [14], [19], [20], [23]).

It is well established that, upon starvation conditions, some randomly located individual $D d$ cells, called pacemakers, begin to secrete periodically a chemical substance (cAMP $\equiv$ cyclic adenosine 3'5'-monophosphate) which propagates in the media in the form of some concentric waves that after some time evolve to a rather peculiar spiral wavelike pattern. This extracellular signal induces receptor modifications in the surrounding cells and activates almost all of the major recognized signalling pathways determining which cells become relay competent, or equivalently, which cells can detect the signal and respond to it by synthesizing and secreting cAMP. Many of the details of the signal transduction/relay process are not known, but an accepted schematic description is the following. After the extracellular cAMP binds to the receptors, the cAMP-receptor complex will activate the adenylate cyclase via G-proteins. The resulting receptor-adenylate cyclase complex catalyzes the conversion of ATP (ATP $\equiv$ adenosine triphosphate) to intracellular cAMP. Intracellular cAMP is hydrolyzed by an intracellular phosphodiesterase or is transported across the plasma membrane into the extracellular medium where it is hydrolyzed by the membrane-bound and extracellular phosphodiesterase. Since cells need a basal intracellular cAMP concentration for normal functions, they begin to secrete cAMP only when the intracellular cAMP concentration exceeds a threshold value (for more details see [6], [15], [20], [22], [23]).

Several models for cAMP propagation, that take into account different aspects of the biochemistry of the problem, have been studied in the scientific literature. Some of the most widely used are Martiel-Goldbeter's model [15], Monk-Othmer's model [16] and Tang-Othmer's model [22]. These models are able to account for the most relevant features of cAMP signals during the cell aggregation process, namely the presence of autonomous oscillations of cAMP, the relay of suprathreshold cAMP pulses, the existence of a refractory period in which cells are unable to answer to additional stimuli. One of these models, namely that proposed in [22], can account 
also for the phenomenon called adaptation, that is the fact that cells react to variations of external cAMP concentration more than to absolute concentrations of external cAMP.

Typically, the systems of equations that have been used to model cAMP signalling contain parameters of different orders of magnitude. We suspect that this does not happen by chance, but that, on the contrary, this should be a common feature of the reaction-diffusion systems describing patterns in biology. Indeed, such patterns are often characterized by the presence of abrupt changes in some physical or chemical magnitudes, or by the presence of structures with very different length scales. It is unlikely that structures with so different scales could be obtained in models that cannot be written in a form containing large or small parameters.

Our goal is to exploit in a systematic manner the scale separation existing in some models for the propagation of cAMP signals. More precisely, in this paper we will focus on the mathematical analysis of the Martiel-Goldbeter model [15]. We will use a similar approach to study also the so-called Goldbeter-Segel model [7]. This latter model seems to be less realistic from the biological point of view, because it assumes that for most parameter values, both the relay response and the oscillations are related with a significant variation of the intracellular ATP induced by the cAMP production, a fact that is ruled out by the experimental results. However, as we shall see, our methods allow to see a rather similar underlying mathematical structure in both problems in spite of the fact that they are based in different biochemical assumptions.

Indeed, after reformulating both the Martiel-Goldbeter and the Goldbeter-Segel models in a manner containing large and small parameters and extending them by including a diffusion term for extracellular cAMP, they can be studied using standard singular perturbation methods. These methods allow to see easily the similar mathematical structure of several models, and on the other hand, they are useful for deriving explicit formulae for magnitudes of interest that hopefully can be compared with experiments and could allow to discriminate between the different models. Moreover, this approach allows to see that some magnitudes which characterize the problems, for instance wave velocity, depend only on a part of the biochemical cycle and can be approximately computed by solving mathematical problems that are simpler than the whole original reaction-diffusion system.

\section{The models: Basic assumptions}

In this Section we summarize the main assumptions on which the Martiel-Goldbeter model and the GoldbeterSegel model are based.

\subsection{Description of the Martiel-Goldbeter model}

In the model proposed by Martiel and Goldbeter [15] (hereafter referred to as the MG model) it is assumed that the cells contain a large number of receptors $\left(10^{5}-10^{6}\right.$ molecules/cell) that might be in either the active or desensitized state. The transition from active to desensitized state takes place by means of the phosphorylation of the cAMP receptor. In the derivation of this model several processes are assumed to play a role inside and outside the cell, namely:

(I) Extracellular cAMP $\left(\mathrm{cAMP}_{\mathrm{e}}\right)$ binds to the receptor. The receptor located on the outer face of the plasma membrane exists in two states, activated $(\mathrm{R})$ and desensitized $(\mathrm{D})$ and the $\mathrm{cAMP}_{\mathrm{e}}$ binds to these two forms with different affinities.

(II) The $\mathrm{cAMP}_{\mathrm{e}}-\mathrm{R}$ complex activates the adenylate cyclase. After diffusion in the membrane, only the form $\mathrm{cAMP}_{\mathrm{e}}-\mathrm{R}$ converts the inactivated adenylate cyclase (IC) into the activated adenylate cyclase (AC), while the form $\mathrm{cAMP}_{\mathrm{e}}-\mathrm{D}$ cannot couple to the cyclase.

(III) Production of intracellular cAMP $\left(\mathrm{cAMP}_{\mathrm{i}}\right)$ from ATP, catalyzed by the AC. The AC has a larger affinity for the substrate ATP and/or a larger catalytic activity than the IC.

(IV) The transition from the active state $(\mathrm{R})$ to the desensitized state $(\mathrm{D})$ of the receptor.

(V) The transport of $\mathrm{cAMP}$ into the extracellular medium.

(VI) cAMP hydrolysis by phosphodiesterase. A part of $\mathrm{CAMP}_{\mathrm{i}}$ is hydrolyzed by the intracellular phosphodiesterase (iPDE), whereas a part is transported in extracellular medium where it is hydrolyzed by the extracellular phosphodiesterase (ePDE).

A scheme for the main steps of the MG model is shown in Figure 2.1. 


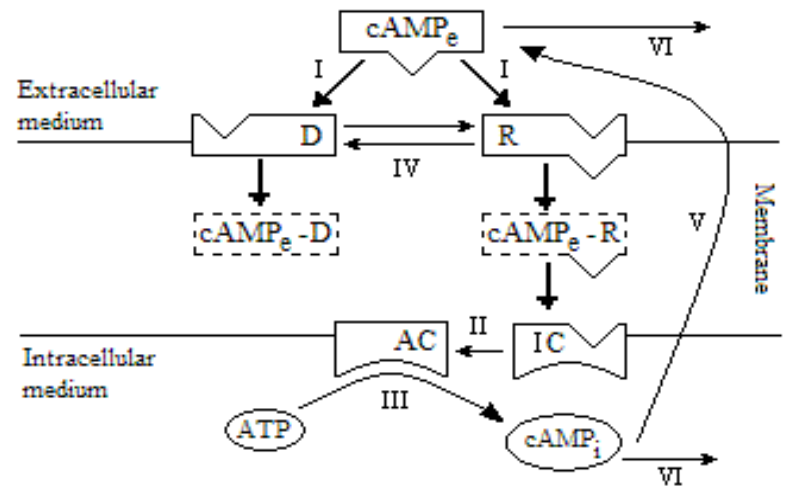

Fig. 2.1: Sketch of the MG model based on receptor desensitization for the cAMP signalling system of the $D d$. The picture is similar to Figure 1 in [15].

All the processes described above are modelled using simple chemical kinetic equations. The resulting problem is a system of nine ODEs that can be reduced to a simpler system containing just three equations using the fact that some of the chemical process are much faster than the others. We refer to the original article [15] for details. The main variables that are used to describe the dynamics of the cAMP signalling system in this model are:

- $\beta$, that represents the concentration of the intracellular cAMP divided by the dissociation constant $K_{R}$;

- $\gamma$, that represents the concentration of the extracellular cAMP divided by the same dissociation constant $K_{R}$;

- $\rho_{T}$, that represents the total fraction of receptors in the active state.

The MG model (cf. [15]), which describes the dynamics of the cAMP signalling system, is characterized by the following set of three ordinary differential equations:

$$
\begin{aligned}
\frac{d \rho_{T}}{d t} & =-f_{1}(\gamma) \rho_{T}+f_{2}(\gamma)\left(1-\rho_{T}\right) \\
\frac{d \beta}{d t} & =q \sigma \phi\left(\rho_{T}, \gamma\right)-\left(k_{i}+k_{t}\right) \beta \\
\frac{d \gamma}{d t} & =\frac{k_{t}}{h} \beta-k_{e} \gamma
\end{aligned}
$$

where the functions $f_{1}(\gamma), f_{2}(\gamma), \phi\left(\rho_{T}, \gamma\right)$ are given by:

$$
\begin{aligned}
& f_{1}(\gamma)=\frac{k_{1}+k_{2} \gamma}{1+\gamma}, \quad f_{2}(\gamma)=\frac{k_{1} L_{1}+k_{2} L_{2} c \gamma}{1+c \gamma} \\
& \phi\left(\rho_{T}, \gamma\right)=\frac{\alpha\left(\lambda \theta+\epsilon Y^{2}\right)}{1+\alpha \theta+\epsilon Y^{2}(1+\alpha)} ; \quad Y=\frac{\rho_{T} \gamma}{1+\gamma}
\end{aligned}
$$

The parameters $k_{i}, L_{i}(i=1,2), c, \alpha, \lambda, \theta, \epsilon$, appearing in the formulae (2.4), (2.5), are as in [15] and their meaning is precised in Tables 1 and 2 . 
Table 1. The meaning of the chemical magnitudes.

\begin{tabular}{|ll|}
\hline Magnitudes & \multicolumn{1}{c|}{ Definition } \\
\hline$k_{1}$ & Rate constant for modification step $\mathrm{R} \rightarrow \mathrm{D}$. \\
$k_{2}$ & Rate constant for modification step cAMP $\mathrm{A} \rightarrow \mathrm{cAMP}_{\mathrm{e}} \mathrm{D}$. \\
$k_{-1}$ & Rate constant for demodification step $\mathrm{D} \rightarrow \mathrm{R}$. \\
$k_{-2}$ & Rate constant for demodification step cAMP $\mathrm{e}_{\mathrm{e}} \mathrm{D} \rightarrow \mathrm{cAMP} \mathrm{e}-\mathrm{R}$. \\
$K_{R}$ & Dissociation constant of cAMP-receptor complex in $\mathrm{R}$ state. \\
$K_{D}$ & Dissociation constant of cAMP-receptor complex in $\mathrm{D}$ state. \\
$K_{m, \text { cyclase }}$ & Michaelis constant of the form AC of adenylate cyclase. \\
$K_{m, \text { cyclase }}^{\prime}$ & Michaelis constant of the form IC of adenylate cyclase. \\
$V_{m, \text { cyclase }}$ & The rate of the maximum adenylate cyclase activity. \\
$k_{i}$ & Apparent first-order rate constant for iPDE. \\
$k_{t}$ & Apparent first-order rate constant for cAMP transport \\
$h$ & into extracellular medium. \\
$V_{m, P D E}$ & Ratio of extracellular to intracellular volumes. \\
$K_{m, P D E}$ & Maximum activity for ePDE. \\
$A T P$ & Michaelis constant of ePDE for cAMP. \\
$k_{5}$ & The intracellular level of ATP. \\
$k_{4}$ & Catalytic constant of the form IC of adenylate cyclase. \\
$\epsilon$ & Catalytic constant of the form AC of adenylate cyclase. \\
\hline
\end{tabular}

Table 2. The expression of the parameters, appearing in (2.1)-(2.5), in terms of the chemical magnitudes of Table 1.

$$
\begin{array}{lll}
L_{1}=k_{-1} / k_{1} & q=K_{m, \text { cyclase }} / K_{R} & \alpha=A T P / K_{m, \text { cyclase }} \\
L_{2}=k_{-2} / k_{2} & \sigma=V_{m, \text { cyclase }} / K_{m, \text { cyclase }} & \theta=K_{m, \text { cyclase }} / K_{m, \text { cyclase }}^{\prime} \\
c=K_{R} / K_{D} & k_{e}=V_{m, P D E} / K_{m, P D E} & \lambda=k_{5} / k_{4}
\end{array}
$$

\subsection{Description of the Goldbeter-Segel model}

In the assumption that the cAMP-receptor on the cell surface is a regulatory part of adenylate cyclase, the Goldbeter and Segel's model [7] (hereafter referred to as the GS model) is a system of ordinary differential equations focusing on the dynamics of extracellular cAMP, intracellular cAMP and autocatalytic regulation of ATP. In this case, both the relay response and the autonomous oscillation of cAMP are related to a significant variation in the substrate ATP induced by the cAMP production. However, such a dependence on the ATP variation seems to be ruled out by the experiments. These show that the concentration of ATP is practically constant during cAMP oscillations (see, for example, [15], [20]). Nevertheless, we shall present in the Appendix B how the methods developed in this paper can be applied also in the case of the GS model.

The main processes that are assumed to play a role in this model are the following:

(I) Extracellular cAMP (cAMP $)$ binds to the receptor.

(II) The $\mathrm{AMP}_{\mathrm{e}}-\mathrm{R}$ complex activates the adenylate cyclase. Upon diffusion in the membrane, the form $\mathrm{cAMP}_{\mathrm{e}^{-}}$ $\mathrm{R}$ converts the inactivated adenylate cyclase (IC) into the activated adenylate cyclase (AC).

(III) Production of intracellular cAMP $\left(\mathrm{cAMP}_{\mathrm{i}}\right)$ from ATP, catalyzed by the AC.

(IV) The transport of cAMP into the extracellular medium.

(V) cAMP hydrolysis by phosphodiesterase. A part of $\mathrm{cAMP}_{\mathrm{i}}$ is hydrolyzed by the intracellular phosphodiesterase (iPDE), whereas a part is transported in extracellular medium where it is hydrolyzed by the extracellular phosphodiesterase (ePDE).

A scheme for the main steps of the GS model is shown in Figure 2.2. 


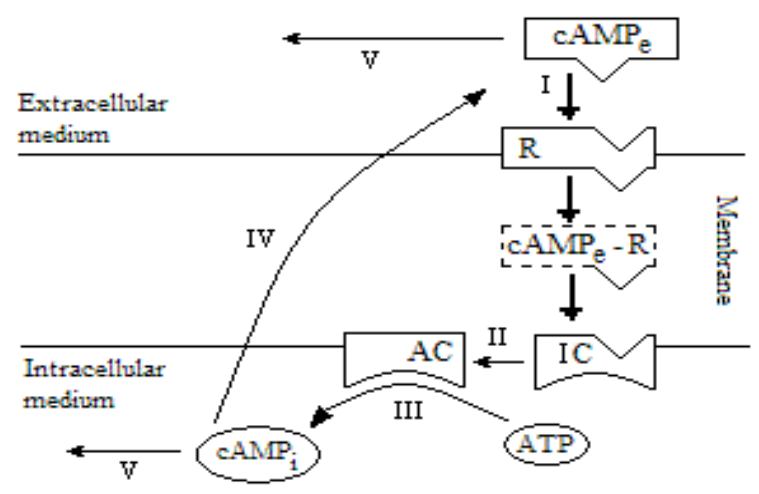

Fig. 2.2: Sketch of the GS model based on the assumption that the cAMP-receptor on the cell surface is a regulatory part of adenylate cyclase. The picture is similar to Figure 1 in [7].

The main variables that are used to describe the dynamics of the cAMP signalling system in this model are:

- $\beta$, that represents the concentration of the intracellular cAMP divided by the dissociation constant $K_{R}$;

- $\gamma$, that represents the concentration of the extracellular cAMP divided by the same dissociation constant $K_{R}$

- $\alpha$, that represents the intracellular level of ATP normalized by the Michaelis constant $K_{m, c y c l a s e}$.

The GS model is then governed by the following three ordinary differential equations:

$$
\begin{aligned}
& \frac{d \alpha}{d t}=v-\sigma \phi(\alpha, \gamma) \\
& \frac{d \beta}{d t}=q \sigma \phi(\alpha, \gamma)-k_{t} \beta \\
& \frac{d \gamma}{d t}=\frac{k_{t}}{h} \beta-k \gamma
\end{aligned}
$$

The rate function $\phi$ for adenylate cyclase is given by:

$$
\phi(\alpha, \gamma)=\frac{\alpha(1+\alpha)(1+\gamma)^{2}}{L+(1+\alpha)^{2}(1+\gamma)^{2}}
$$

and $v$ denotes a constant ATP input rate divided by the Michaelis constant $K_{m, c y c l a s e}, k$ measures the assumed linear rate at which extracellular cAMP is destroyed by the membrane-bound and extracellular forms of phosphodiesterase and $L$ denotes the allosteric constant of adenylate cyclase. The other parameters appearing in (2.6)-(2.8), $\sigma, q, k_{t}$, and $h$, are those given in Table 2.

\section{Orders of magnitude in the model}

\subsection{Some additional biochemical assumptions}

Our goal is to study the propagation of cAMP pulses for a system of PDEs having as underlying kinetics the set of equations (2.1)-(2.3). We shall assume that the extracellular cAMP diffuses according to the standard Fick's law. We are then led to study the following system of equations:

$$
\begin{aligned}
\frac{\partial \rho_{T}}{\partial t} & =-f_{1}(\gamma) \rho_{T}+f_{2}(\gamma)\left(1-\rho_{T}\right) \\
\frac{\partial \beta}{\partial t} & =q \sigma \phi\left(\rho_{T}, \gamma\right)-\left(k_{i}+k_{t}\right) \beta \\
\frac{\partial \gamma}{\partial t} & =\frac{k_{t}}{h} \beta-k_{e} \gamma+D \frac{\partial^{2} \gamma}{\partial x^{2}}
\end{aligned}
$$


where $D$ is the diffusion coefficient, and $f_{1}(\gamma), f_{2}(\gamma), \phi\left(\rho_{T}, \gamma\right)$ are as in $(2.4),(2.5)$.

We wish to reformulate (3.1)-(3.3) as a singular perturbation problem. Moreover, we are interested in the travelling wave regime associated to (3.1)-(3.3). A set of values of the parameters that has been shown in [15] to yield typical travelling wave behaviour is given in Table 3.

Table 3. The numerical values of the parameters considered in the model.

\begin{tabular}{|c|c|c|c|}
\hline $\begin{array}{l}\text { Adimensional } \\
\text { parameter }\end{array}$ & $\begin{array}{l}\text { Numerical } \\
\text { value }\end{array}$ & Parameter & $\begin{array}{l}\text { Numerical } \\
\text { value }\end{array}$ \\
\hline$L_{1}$ & 10 & $k_{1}$ & $0.4 \mathrm{~min}^{-1}$ \\
\hline$L_{2}$ & 0.1 & $k_{2}$ & $0.004 \mathrm{~min}^{-1}$ \\
\hline$c$ & 100 & $\sigma$ & $0.57 \mathrm{~min}^{-1}$ \\
\hline$q$ & $4 \times 10^{3}$ & $k_{i}$ & $1 \min ^{-1}$ \\
\hline$h$ & 5 & $k_{t}$ & $0.9 \mathrm{~min}^{-1}$ \\
\hline$\alpha$ & 3 & $k_{e}$ & $4 \min ^{-1}$ \\
\hline$\lambda$ & 0.01 & & \\
\hline$\theta$ & 0.01 & & \\
\hline$\epsilon$ & 0.15 & & \\
\hline
\end{tabular}

Following [15], we remark that, if the transition between the two conformational states of the receptors $R$ and $D$ is just a simple conformational change, the microscopic reversibility condition holds:

$$
L_{1}=c L_{2}
$$

We notice that (3.4) will not be satisfied in general if the transition between the two states of the receptors must be represented by a process of covalent modification. Nevertheless, we will assume in the rest of the paper that (3.4) is valid. We define

$$
\mathcal{P}=\frac{k_{1}}{k_{2}}, \quad d=\frac{1+L_{1}}{c+L_{1}}
$$

and we introduce a new time scale by means of:

$$
\tau=\left(k_{i}+k_{t}\right) t
$$

Plugging the formulae (2.4), (2.5) into the equations (3.1)-(3.3) and using (3.4), (3.5) and (3.6) we obtain, after rearranging some terms:

$$
\begin{aligned}
\frac{\partial \rho_{T}}{\partial \tau} & =\frac{k_{2}}{\left(k_{i}+k_{t}\right)} \frac{c\left(1+L_{2}\right)}{1+c \gamma} \cdot(\gamma+\mathcal{P})\left[\frac{L_{2}}{\left(1+L_{2}\right)}-\frac{\gamma+d}{\gamma+1} \rho_{T}\right] \\
\frac{\partial \beta}{\partial \tau} & =\frac{\alpha q \sigma}{(1+\alpha)\left(k_{i}+k_{t}\right)} \cdot \frac{\frac{\lambda \theta}{\epsilon}(1+\gamma)^{2}+\left(\rho_{T} \gamma\right)^{2}}{\frac{(1+\alpha \theta)}{(1+\alpha) \epsilon}(1+\gamma)^{2}+\left(\rho_{T} \gamma\right)^{2}}-\beta \\
\frac{\partial \gamma}{\partial \tau} & =\frac{k_{e}}{k_{i}+k_{t}}\left(\frac{k_{t}}{k_{e} h} \beta-\gamma\right)+\frac{D}{k_{i}+k_{t}} \cdot \frac{\partial^{2} \gamma}{\partial x^{2}}
\end{aligned}
$$

\subsection{A rough estimate of the orders of magnitude of the variables}

As a first step, in order to get some intuition on the form of the solutions of (3.7)-(3.9), we make a comparison between the relative sizes of the terms arising there. We shall use repeatedly the asymptotic notations $\ll, ~$ and $\simeq$ (see Appendix A for the precise definitions).

Let us consider spatially homogeneous solutions of (3.7)-(3.9). Therefore, the diffusive term in (3.9) will be ignored.

The numerical values in Table 3 give $k_{i}+k_{t}=1.9 \mathrm{~min}^{-1}, k_{e}=4 \mathrm{~min}^{-1}$. This suggests that the characteristic time for approaching equilibrium is twice for $\beta$ than for $\gamma$ (cf. (3.8), (3.9)). In particular, this indicates that the evolution of the third equation is roughly adiabatic during the time scales in which $\beta$ is far from equilibrium, i.e. the equation (3.9) might be replaced approximately by the quasi-steady-state condition

$$
\frac{k_{t}}{k_{e} h} \beta-\gamma=0, \quad \text { i.e. } \quad \beta=\frac{k_{e} h}{k_{t}} \gamma
$$


We remark that this heuristic assumption is very rough and we are using it just to estimate the order of magnitude of the variables $\rho, \beta, \gamma$. We shall derive later a singular perturbation problem having "true" small parameters. In particular, we are not claiming here that the "small parameter" in the problem is the quotient of the characteristic times of (3.9) and (3.8), which is just of order 0.5 for the numerical values of the parameters as in Table 3. Let us notice, however, that although the assumption (3.10) is very rough, it is approximately satisfied in the numerical simulations (see Figure 3.1).
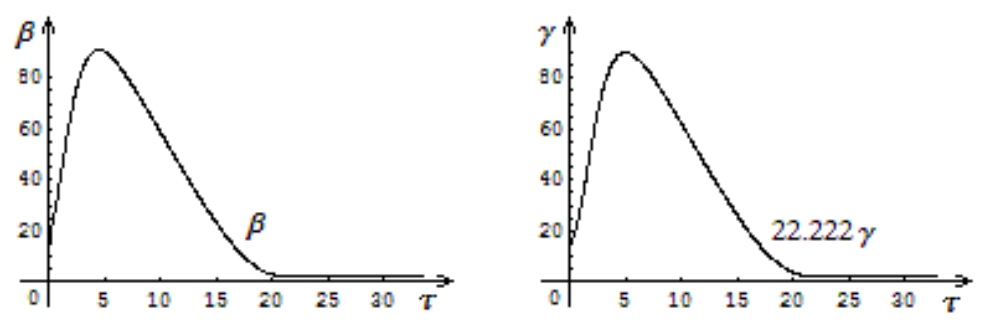

Fig. 3.1: Relay response to cAMP pulses. These curves have been obtained by numerical integration of the three-variable system (3.7)-(3.9), for homogeneous solutions, with the numerical value of the parameters as in Table 3. The values of the initial conditions are above the threshold value. The concentration of cAMP, $\gamma$, in this picture is multiplied by the factor $\frac{k_{e} h}{k_{t}} \approx 22.222$.

Using the approximation (3.10) and introducing the notations

$$
b=\frac{\alpha q \sigma k_{t}}{\left(k_{i}+k_{t}\right) k_{e} h} \cdot \frac{\lambda \theta}{1+\alpha \theta}, \quad B=\frac{\alpha q \sigma k_{t}}{\left(k_{i}+k_{t}\right) k_{e} h} \cdot \frac{1}{1+\alpha},
$$

the signalling system (3.7)-(3.9) reduces, in the spatially homogeneous case, to the following two-variables system:

$$
\begin{aligned}
\frac{d \rho_{T}}{d \tau} & =\frac{k_{2}}{\left(k_{i}+k_{t}\right)} \cdot \frac{c\left(1+L_{2}\right)}{1+c \gamma} \cdot(\gamma+\mathcal{P})\left[\frac{L_{2}}{1+L_{2}}-\frac{\gamma+d}{\gamma+1} \rho_{T}\right] \\
\frac{d \gamma}{d \tau} & =\frac{b \frac{(1+\alpha \theta)}{(1+\alpha) \epsilon}(1+\gamma)^{2}+B\left(\rho_{T} \gamma\right)^{2}}{\frac{(1+\alpha \theta)}{(1+\alpha) \epsilon}(1+\gamma)^{2}+\left(\rho_{T} \gamma\right)^{2}}-\gamma
\end{aligned}
$$

As indicated before, the reduction of (3.7)-(3.9) to the two-variables system (3.12)-(3.13) is not rigorously justified. Nevertheless, the numerical integration of the equations (3.12)-(3.13) shows that this provides a reasonable approximation of the three-variable system (3.7)-(3.9) for spatially homogeneous solutions (see Figure $3.2)$. The advantage of the reduction of (3.7)-(3.9) to the two-variable system (3.12)-(3.13) is that the last one can be studied in the phase plane.
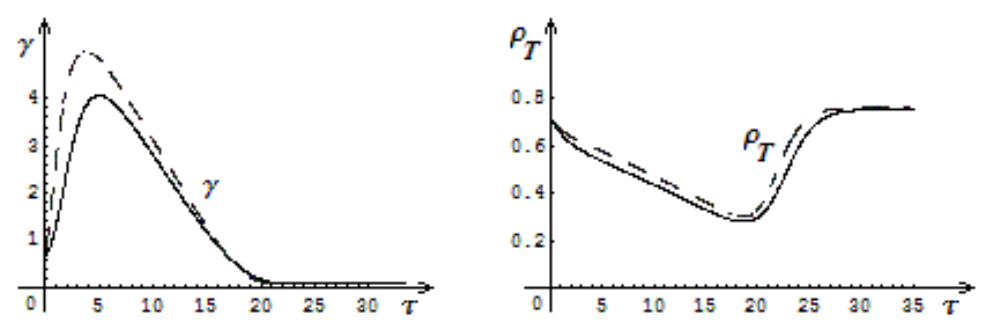

Fig. 3.2: Comparison of relay behaviour in the two variable system (3.12)-(3.13) (dashed lines) and in the threevariable system (3.7)-(3.9) (solid lines). The curves are obtained for the numerical values of the parameters as in Table 3, and with values of the initial conditions above the threshold value. 
The nullclines of the system (3.12)-(3.13) are the curves:

$$
\begin{aligned}
\frac{d \rho_{T}}{d \tau} & =0 \Rightarrow \rho_{T}=\frac{L_{2}}{1+L_{2}} \cdot \frac{\gamma+1}{\gamma+d} \\
\frac{d \gamma}{d \tau} & =0 \Rightarrow \rho_{T}=\sqrt{\frac{(1+\alpha \theta)}{(1+\alpha) \epsilon}} \cdot \frac{\gamma+1}{\gamma} \cdot \sqrt{\frac{\gamma-b}{B-\gamma}}
\end{aligned}
$$

which are represented in Figure 3.3 and, respectively, Figure 3.4 for the numerical values of the parameters as in Table 3. The nullcline $\frac{d \gamma}{d \tau}=0$ is of "cubic" shape. By this we mean that for $\rho_{T}$ in the suitable interval, there are three solutions $\gamma=\gamma\left(\rho_{T}\right)$ of the equation $\frac{d \gamma}{d \tau}=0$.

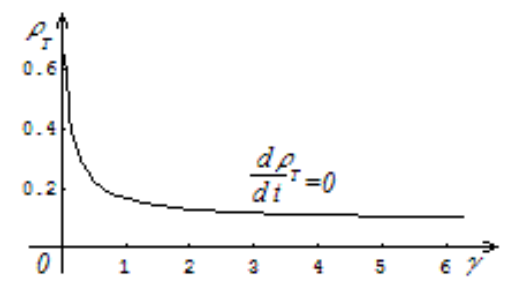

Fig. 3.3: The nullcline $\frac{d \rho_{T}}{d \tau}=0$ corresponding to the system (3.12)-(3.13). The curve is obtained for the numerical values of the parameters as in Table 3 . It represents a monotonically decreasing function approaching to the constant value $\frac{L_{2}}{1+L_{2}}$ as $\gamma \rightarrow \infty$.
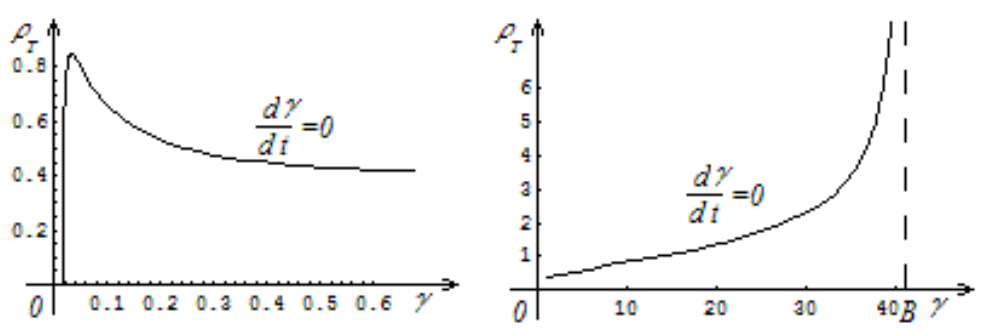

Fig. 3.4: The nullcline $\frac{d \gamma}{d \tau}=0$ corresponding to the system (3.12)-(3.13). The curve is obtained for the numerical values of the parameters as in Table 3. It has a "cubic" shape. The same figure is plotted using two different scales because the region close to the origin will be particularly relevant.

It is easy to see that, in the phase plane $\left(\rho_{T}, \gamma\right)$, the nullclines have only a intersection point, i.e. a steady state, situated on the leftmost branch of the curve $\frac{d \gamma}{d \tau}=0$, but close to the local maximum, like in Figure 3.5.
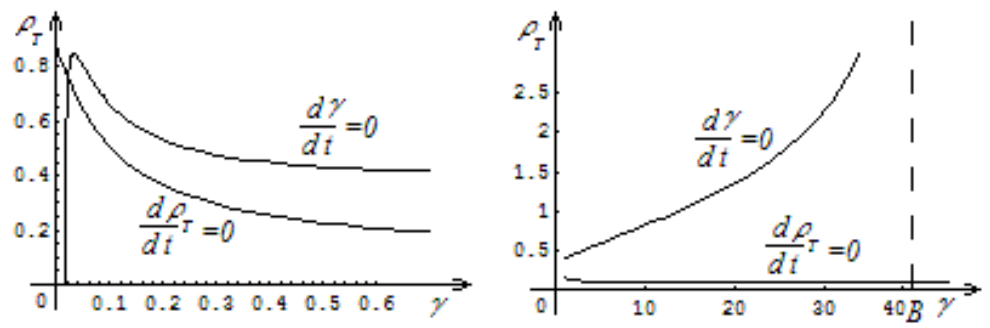

Fig. 3.5: The nullclines corresponding to the system (3.12)-( 3.13) have only one intersection point $\left(\gamma, \rho_{T}\right) \approx$ $(0.0221,0.762)$. The curves are obtained for the numerical values of the parameters as in Table 3 . The system admits a single, stable steady state. 
The system (3.12)-(3.13) has several analogies and also some differences with the underlying kinetics associated to the well known FitzHugh Nagumo model (hereafter referred to as the FHN model), that has been repeatedly used as a first approximation of the dynamics of excitable systems ([8], [11], [18], [21]). The kinetics of such a system is characterized by the fact that the two variables in the model change in different time scales. Moreover, for each value of the slow variable, the fast variable admits three steady states in the relevant regime, one of them unstable and the two others stable. Notice that, since $\frac{k_{2}}{\left(k_{i}+k_{t}\right)} \approx 0.0021$ is rather small, the variable that will play the role of the fast variable in (3.12)-(3.13) is $\gamma$.

In order to obtain a rough description of the dynamics of (3.12)-(3.13), we can then argue as in the classical study of the FHN model. For each fixed value of $\rho_{T}$ in a suitable range, there are three positive roots of (3.15). For the dynamics given by the equation (3.13), two of these roots yield stable behaviours and the root in the intermediate position is unstable. Therefore, the system (3.12)-(3.13) is excitable, which means that a perturbation from the steady state $\left(\rho_{T, S}, \gamma_{S}\right)$ to a point in the phase plane $\left(\rho_{T}, \gamma\right)$ having a value of $\gamma$ larger than the intermediate root of (3.15) for each given value of $\rho_{T}$, initiates a large excursion before returning to the steady state. In other words, this intermediate root of (3.15) is the threshold beyond which perturbations of the steady state of (3.12)-(3.13) move away from it.

The main difference between the system (3.12)-(3.13) and the classical FHN model is the smallness of the threshold, an assumption that is not usually made in the analysis of the FHN model.

\subsection{Some restrictions on the parameters in order to recover the nullclines struc- ture}

Our next goal is to define a particular distinguished limit of (3.7)-(3.9) that could describe the main features observed for the solutions, if the numerical values of the parameters are as in Table 3. In particular, we would like to keep the analogies with the FHN model that we have explained in the previous Subsection.

In order to obtain an asymptotic limit preserving the features of the solutions described in the Subsection 3.2 , we need to impose some constraints an the parameters appearing in (3.7)-(3.9). More precisely, we would like the curves defined in (3.14) and (3.15) to have the same shape as in the Figures 3.3 and 3.4, respectively. Moreover, we would like to have only one homogeneous steady state. This is just the intersection point of the curves (3.14), (3.15) situated on the left of the local maximum of the curve (3.15), but with $\rho_{T}$ having the same order of magnitude as in that local maximum, as in Figure 3.5. We denote:

$$
A=\frac{(1+\alpha \theta)}{(1+\alpha) \epsilon} \cdot\left(\frac{1+L_{2}}{L_{2}}\right)^{2} .
$$

Some tedious, but straightforward algebra, shows that the nullclines of the system (3.12)-(3.13) satisfy all the previously requirements if we assume:

$$
\begin{gathered}
1 \simeq 4 b B<A(2 b+d)^{2}<4 b B+b B\left(\frac{b+d}{2 b+d}\right)^{2} \\
3 b \ll 2 d-b<\frac{B}{A}<2 d-b+\sqrt{3 d(d-2 b)}<1
\end{gathered}
$$

Notice that for the numerical values of the parameters as in Table 3 we have $\frac{k_{e}}{k_{i}+k_{t}} \approx 2.1$. Therefore, we shall make the assumption

$$
\frac{k_{e}}{k_{i}+k_{t}} \simeq 1
$$

Finally, since $\frac{k_{2}}{k_{i}+k_{t}} \approx 0.0021$, it is natural to impose:

$$
\frac{k_{2}}{k_{i}+k_{t}} \ll 1
$$

It is readily seen that the conditions $(3.17),(3.18)$ are satisfied by the numerical values of the parameters as in Table 3. Therefore, the asymptotic limit (3.17)-(3.20) captures the dynamics of the system (3.7)-(3.9) for the values of the parameters as in Table 3. In the remainder of this paper we shall study the mathematical properties of this limit.

In Figure 3.6 we show which is the aspect of the nullclines (3.14), (3.15) in the asymptotic limit (3.17)-(3.20). 


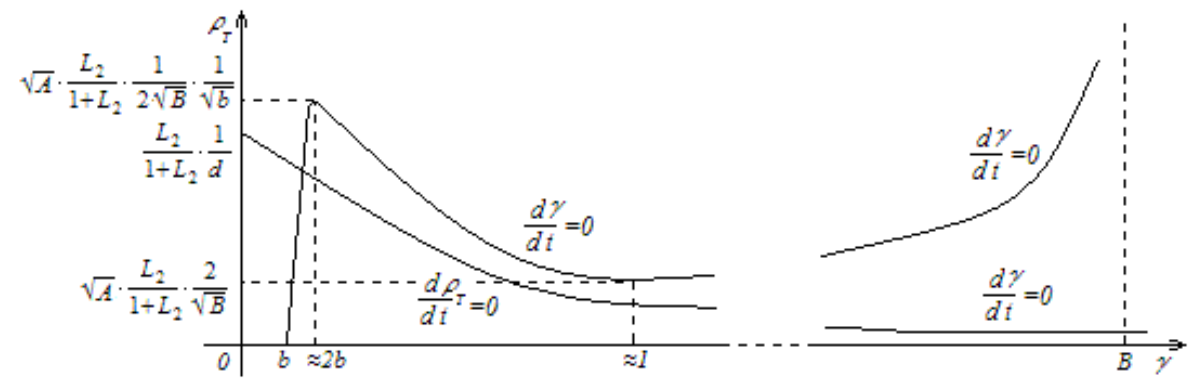

Fig. 3.6: The nullclines corresponding to the system (3.12)-(3.13) in the hypotheses (3.17)-(3.20).

\subsection{Formulating the singular perturbation problem}

We shall study the system (3.7)-(3.9) in the asymptotic limit (3.17)-(3.20). As a first step, we introduce a suitable set of adimensional variables.

The unique spatially homogeneous steady state of (3.7)-(3.9) is given by

$$
\left(\rho_{T, S}, \beta_{S}, \gamma_{S}\right)=\left(\frac{L_{2}(m+1)}{\left(1+L_{2}\right)(m+d)}, \frac{k_{e} h}{k_{t}} m, m\right)
$$

where $m$ is the unique positive root of the equation $A(\gamma-b)(\gamma+d)^{2}-\gamma^{2}(B-\gamma)=0$. We notice that the order of magnitude of this root in the asymptotic limit (3.17)-(3.20) is

$$
b<m \lesssim 2 b .
$$

Due to the fact that we expect to have a dynamics for the system (3.7)-(3.9) analogous to the one of the FHN system (cf. Subsection 3.2) and also because $\gamma$ is the fast variable, we might expect that during the excursions of the solutions of the system far from the steady state, the maximum value of $\gamma$ is given by the largest root of (3.15) with $\rho_{T}=\rho_{T, S}$. Taking into account (3.21), it follows that such a root is the largest one of the equation $A(\gamma-b)(\gamma+1)^{2}-\frac{(m+1)^{2}}{(m+d)^{2}} \gamma^{2}(B-\gamma)=0$. We shall denote this root $\gamma=M$. In the distinguished limit (3.17)-(3.20) the asymptotic behaviour of $M$ is:

$$
M \simeq \frac{1}{8 b}
$$

We shall take as characteristic values for the variables $\rho, \beta, \gamma$ the magnitudes $\rho_{T, S}, \frac{k_{e} h}{k_{t}} M, M$. The choice for $\beta$ is due to (3.10). The analogy with the FHN system suggests that these characteristic values are roughly the maximum values of $\rho, \beta, \gamma$ during their evolution. Therefore, in order to obtain a system of equations whose variables are at most of order one, we introduce adimensional variables $R, W, U$ by means of

$$
\begin{aligned}
\rho_{T} & =\rho_{T, S} \cdot R \\
\beta & =\frac{k_{e} h}{k_{t}} M \cdot W \\
\gamma & =M \cdot U
\end{aligned}
$$

On the other hand, (3.9) suggests to introduce an adimensional space variable $\widetilde{x}$ by means of

$$
x=\sqrt{\frac{D}{k_{i}+k_{t}}} \cdot \widetilde{x}
$$

Using the previous changes of variables, the system (3.7)-(3.9) becomes:

$$
\begin{aligned}
& R_{\tau}(\widetilde{x}, \tau)=\kappa(U+\mathcal{P} \varepsilon)\left[\frac{\mu(U+\varepsilon)-(U+d \varepsilon) R}{\left(U+\frac{\varepsilon}{c}\right)(U+\varepsilon)}\right] \\
& W_{\tau}(\widetilde{x}, \tau)=\frac{b \varepsilon(U+\varepsilon)^{2}+\Theta R^{2} U^{2}}{(U+\varepsilon)^{2}+\Lambda R^{2} U^{2}}-W \\
& U_{\tau}(\widetilde{x}, \tau)=U_{\widetilde{x} \widetilde{x}}+\Gamma[W-U]
\end{aligned}
$$

where the parameters are given in Table 4, that contains also their orders of magnitude and the numerical values. 
Table 4. The orders of magnitudes and the numerical value of the parameters of (3.25)-(3.27).

\begin{tabular}{|clcc|}
\hline $\begin{array}{c}\text { Parameters of } \\
(3.25)-(3.27)\end{array}$ & \multicolumn{1}{c}{ Definition } & $\begin{array}{c}\text { Orders of } \\
\text { magnitudes }\end{array}$ & $\begin{array}{c}\text { Numerical value } \\
\text { using Table } 3\end{array}$ \\
\hline$\kappa$ & $\frac{k_{2}\left(1+L_{2}\right)}{k_{i}+k_{t}}$ & $\ll 1$ & 0.0023 \\
$\mu$ & $\frac{m+d}{m+1}$ & $\ll 1$ & 0.1274 \\
$\varepsilon$ & $M^{-1} \simeq 8 b$ & $\ll 1$ & 0.1258 \\
$d$ & $\frac{1+L_{1}}{c+L_{1}}$ & $\ll 1$ & 0.1 \\
$b$ & $\frac{\alpha q \sigma k_{t}}{\left(k_{i}+k_{t}\right) k_{e} h} \cdot \frac{\lambda \theta}{1+\alpha \theta}$ & $\ll 1$ & 0.0157 \\
$\Gamma$ & $\frac{k_{e}}{k_{i}+k_{t}}$ & $\simeq 1$ & 2.1052 \\
$\Lambda$ & $\left(A \mu^{2}\right)$ & $\simeq 1$ & 0.2966 \\
$\Theta$ & $\Lambda B \varepsilon$ & $\simeq 1$ & 1.5087 \\
$c$ & & $\gg 1$ & 100 \\
$\mathcal{P}$ & $\frac{k_{1}}{k_{2}}$ & $\gg 1$ & 100 \\
$\kappa \mathcal{P}$ & $\frac{k_{1}\left(1+L_{2}\right)}{k_{i}+k_{t}}$ & $\simeq 1$ & 0.2315 \\
$\mathcal{P} \varepsilon$ & $\frac{k_{1}}{M k_{2}}$ & $\simeq 1$ or $\gg 1$ & 12.582 \\
\hline
\end{tabular}

We now discuss why the orders of magnitude are chosen as in Table 4. We notice that in the asymptotic limit (3.17)-(3.20) we have

$$
b \ll 1 \ll B
$$

due to (3.17) and (3.18) and so, using the definition of $\varepsilon$ given in Table 4 , this implies $\varepsilon \ll 1$.

We now proceed to estimate the order of magnitude of the remaining parameters in Table 4. Due to (3.18), (3.22) and (3.28), we have

$$
\mu \sim d
$$

and so, using the definition of $\Lambda$ given in Table 4, we have $\Lambda \sim\left(A d^{2}\right)^{-1}$. From (3.17) it then follows that $\Lambda \simeq 1$. Therefore, using again (3.17) and the definitions of $\Theta$ and $\varepsilon$ given in Table 4 , we obtain $\Theta \simeq 1$.

Using the fact that $A \gg 1$ (cf. (3.18), (3.28)), as well as the definition of $\Lambda$, it follows that $\mu \ll 1$, whence $d \ll 1$. Using the definition of $d$ in (3.5) and (3.4), it follows that

$$
c \gg \max \left\{1, L_{1}\right\}, \quad L_{2} \ll 1
$$

whence

$$
d \gg \frac{1}{c}
$$

The biochemical interpretation of the fact that $c \gg 1$ is that the receptor possesses a high-affinity desensitized state and the fact that $L_{2}<1$ means that the form $\mathrm{cAMP}_{\mathrm{e}}-\mathrm{D}$ predominates at ligand saturation. Let us make the remark that if $L_{1}>1$, this means that the activated state of the receptor $(\mathrm{R})$ predominates in the absence of ligand.

On the other hand, by (3.17), (3.18) and (3.29), we have

$$
\mu \simeq \varepsilon
$$

Combining (3.20) with (3.30), it follows that $\kappa \ll 1$. Finally, notice that (3.19) implies that $\Gamma \simeq 1$.

Concerning the parameter $\mathcal{P} \varepsilon$, we remark that the analysis carried on in the next pages can be made without major differences both in the cases of $\mathcal{P} \varepsilon \simeq 1$ or $\mathcal{P} \varepsilon \gg 1$.

One can notice that the numerical values of the parameters which appear in system (3.25)-(3.27), obtained taking into account Table 3, are consistent with the distinguished limit defined in Table 4.

We remark that the derivation of the asymptotic limit defined in Table 4 is not a rigorous mathematical argument. It is a combination of the constraints on the parameters formulated in Subsection 3.3 with some ansatz on the asymptotic limit using the numerical values of the parameters of the problem.

\section{Mathematical analysis: travelling waves}

This Section is devoted to the study of travelling wave solutions of the system (3.25)-(3.27) under the assumptions considered in Table 4 . The reason for expecting the existence of travelling waves for this system is the 
usual one in excitable systems: the diffusion effects induce propagating waves of excitation in spatial direction. So, once an excitation is given that lies above the threshold at a spatial point, the diffusion effect forces neighbouring points to rise above the threshold, causing $U$ to be excited, and the excitation propagates. Thus, the wave propagates by successive neighbourhoods stimulating one another (via the diffusion in the fast variable $U)$ to become superthreshold, whereupon the dynamics begin to dominate. However, since there is a unique steady state for the system, in a longer time scale the variables describing the system approach equilibrium on the rear of the wave. The goal of this section is to describe all these processes in analytical manner.

To begin with, we look for solutions of (3.25)-(3.27) of the form

$$
R(\widetilde{x}, \tau)=R(\xi), \quad W(\widetilde{x}, \tau)=W(\xi), \quad U(\widetilde{x}, \tau)=U(\xi)
$$

setting $\xi=\widetilde{x}-\mathcal{V}_{\tau}$, where the wave speed $\mathcal{V}>0$ is to be determined in the course of the analysis $(\mathcal{V}$ depends on the parameters of the system). Plugging (4.1) into the system (3.25)-(3.27), we readily see that this becomes:

$$
\begin{aligned}
& -\mathcal{V} R_{\xi}(\xi)=\kappa(U+\mathcal{P} \varepsilon)\left[\frac{\mu(U+\varepsilon)-(U+d \varepsilon) R}{\left(U+\frac{\varepsilon}{c}\right)(U+\varepsilon)}\right] \\
& -\mathcal{V} W_{\xi}(\xi)=\frac{b \varepsilon(U+\varepsilon)^{2}+\Theta R^{2} U^{2}}{(U+\varepsilon)^{2}+\Lambda R^{2} U^{2}}-W \\
& -\mathcal{V} U_{\xi}(\xi)=U_{\xi \xi}+\Gamma[W-U]
\end{aligned}
$$

We are interested in finding, for some particular choice of $\mathcal{V}$, pulse-like solutions satisfying the following limit conditions:

$$
\lim _{|\xi| \rightarrow \infty} R(\xi)=R_{s}, \quad \lim _{|\xi| \rightarrow \infty} W(\xi)=W_{s}, \quad \lim _{|\xi| \rightarrow \infty} U(\xi)=U_{s}
$$

where $\left(R_{s}, W_{s}, U_{s}\right) \simeq(1, b \varepsilon, b \varepsilon)$ are the values of the concentrations for the unique spatially homogeneous equilibrium state of (3.25)-(3.27).

Since the problem (4.2)-(4.5) contains small parameters $(0<\kappa \ll 1,0<\varepsilon \ll 1,0<\mu \ll 1,0<d \ll 1$, $\left.0<\frac{1}{c} \ll 1,0<b \ll 1\right)$ it is possible to study it using a singular perturbation approach. This means that we can "split" the initial system into reduced "fast" and "slow" subsystems. We want to obtain a solution of (4.2)-(4.5), such that the $U$-component behaves as indicated in the Figure 4.1.

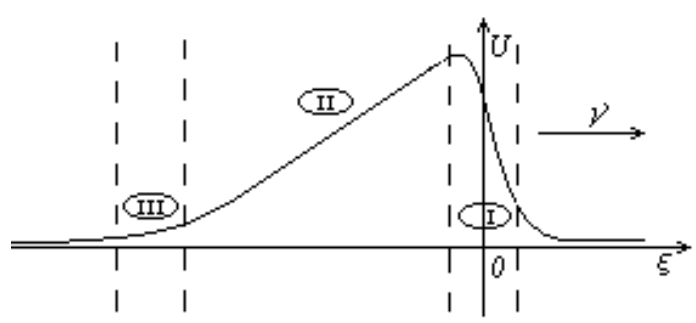

Fig. 4.1: The $U$-component of a pulse solution of the system (4.2)-(4.5). The parts of the pulse are denoted as I (the leading front), II (the top of the pulse) and III (the trailing front).

An asymptotic singular solution of the initial system, in the limit considered in Table 4, consists of several pieces, each piece being a solution of one of the reduced subsystems. The complete solution requires determining the wave speed $\mathcal{V}$ and the parts of the pulse (the leading front, the top and the trailing front) making sure that they all join up consistently (see Figure 4.1). To get this, we have to carry out a singular perturbation analysis, the upshot of which gives the behaviour of the variables $R, W$ and $U$.

\subsection{The leading front (the fast transition)}

The first part of the pulse consists in the arrival of a diffusive front to a quiescent region. As a consequence, the variables $W$ and $U$ become larger than the threshold of excitability and they jump rather quickly to a larger characteristic value of the excited state. Taking into account that $\kappa P \simeq 1, \kappa \ll 1$ and $\varepsilon \ll 1$ (cf. Table 4 ), it follows that during this part of the process the value of $R$ remains approximately constant. Indeed, this 
assumption implies that, for $W$ and $U$ of order one, the right hand side of the equation (4.2) is much smaller than the right hand side of the equations (4.3)-(4.4). Therefore, in the description of the leading front we will assume that the value of $R$ remains frozen as $R=R_{s}$. Henceforth, (4.3)-(4.4) reduces in this region to

$$
\begin{aligned}
& -\mathcal{V} W_{\xi}(\xi)=\frac{b \varepsilon(U+\varepsilon)^{2}+\Theta R_{s}^{2} U^{2}}{(U+\varepsilon)^{2}+\Lambda R_{s}^{2} U^{2}}-W \\
& -\mathcal{V} U_{\xi}(\xi)=U_{\xi \xi}+\Gamma[W-U]
\end{aligned}
$$

The equations (4.6) and (4.7) describe the travelling wave solutions associated to the reaction-diffusion system

$$
\begin{aligned}
W_{\tau}(\widetilde{x}, \tau) & =\frac{b \varepsilon(U+\varepsilon)^{2}+\Theta R_{s}^{2} U^{2}}{(U+\varepsilon)^{2}+\Lambda R_{s}^{2} U^{2}}-W \\
U_{\tau}(\widetilde{x}, \tau) & =U_{\widetilde{x} \widetilde{x}}+\Gamma[W-U]
\end{aligned}
$$

This last system has three homogeneous steady states, $\left(W_{1}, U_{1}\right)$, $\left(W_{2}, U_{2}\right)$, $\left(W_{3}, U_{3}\right)$, where $U_{1}<U_{2}<U_{3}$ and $U_{i}=W_{i}, i=1,2,3$, as long as $R_{s}$ remains of order one. The steady states $\left(W_{1}, U_{1}\right) \simeq(b \varepsilon, b \varepsilon)$ and $\left(W_{3}, U_{3}\right) \simeq(a, a)$ are linearly stable, where

$$
a=\frac{\Theta R_{s}^{2}}{1+\Lambda R_{s}^{2}} \simeq 1
$$

and the steady state $\left(W_{2}, U_{2}\right)$ is unstable. Therefore, we must consider the system (4.6)-(4.7) with the conditions

$$
\begin{array}{ll}
\lim _{\xi \rightarrow-\infty} U(\xi)=U_{3}, & \lim _{\xi \rightarrow-\infty} W(\xi)=W_{3} \\
\lim _{\xi \rightarrow+\infty} U(\xi)=U_{1}, & \lim _{\xi \rightarrow+\infty} W(\xi)=W_{1}
\end{array}
$$

The system takes different forms for $U \gg \varepsilon$ or $U \simeq \varepsilon$ as $\varepsilon \rightarrow 0$. We shall study these different regions separately.

\subsubsection{Region I $(U \gg \varepsilon)$}

To begin with, we assume $U \gg \varepsilon$. In this region, letting $\varepsilon \rightarrow 0$, the system (4.6)-(4.7) can be approximated by

$$
\begin{aligned}
-\mathcal{V} W_{\xi} & =a-W \\
-\mathcal{V} U_{\xi} & =U_{\xi \xi}+\Gamma[W-U]
\end{aligned}
$$

where $a$ is given in (4.8). On the other hand, the asymptotics on $\left(W_{1}, U_{1}\right)$ and $\left(W_{3}, U_{3}\right)$ allow us to rewrite the boundary conditions (4.9)-(4.10) as

$$
\begin{array}{ll}
\lim _{\xi \rightarrow-\infty} U(\xi)=a, & \lim _{\xi \rightarrow-\infty} W(\xi)=a \\
\lim _{\xi \rightarrow+\infty} U(\xi)=0, & \lim _{\xi \rightarrow+\infty} W(\xi)=0
\end{array}
$$

The problem (4.11)-(4.13) is linear and it can be explicitly solved. Its solution is given by:

$$
\begin{aligned}
W(\xi) & =a-C e^{\frac{\xi}{\mathcal{V}}} \\
U(\xi) & =a+C \frac{\Gamma \mathcal{V}^{2}}{1+\mathcal{V}^{2}(1-\Gamma)} e^{\frac{\xi}{\mathcal{V}}}+K e^{\frac{1}{2}\left(-\mathcal{V}+\sqrt{\mathcal{V}^{2}+4 \Gamma}\right) \xi}
\end{aligned}
$$

where $C$ and $K$ are arbitrary constants which will be determined and $1+\mathcal{V}^{2}(1-\Gamma) \neq 0$. Note that the solution given by (4.15)-(4.16) satisfies the boundary condition (4.13). For $U(\xi)$ to represent the leading front of a pulse we must have $C>0$.

Notice that we cannot expect the solutions of the system (4.11)-(4.13) to satisfy the boundary condition (4.14) because, for $\xi \rightarrow+\infty, U$ becomes of order $\varepsilon$ or smaller and this system will be not valid there. In that region, we shall need to use the whole system (4.6)-(4.7), introducing some suitable boundary layer.

In order to have the condition (4.14) satisfied, the function $U$ must become of order $\varepsilon$ for some value of $\xi$. Since in the derivation of the problem (4.11)-(4.14) we made $\varepsilon \rightarrow 0$, this means that the function $U$ defined in (4.16) must vanish at some point $\xi=\xi_{0}$. Therefore, $U\left(\xi_{0}\right)=0$ and $U^{\prime}\left(\xi_{0}\right) \leqslant 0$. Actually, it turns out that $U^{\prime}\left(\xi_{0}\right)=0$, 
at the limit $\varepsilon \rightarrow 0$. Indeed, otherwise $U$ would become negative in the neighbourhood of $\xi=\xi_{0}$ (since $U$ is the solution of the equation (4.7), and $W$ is bounded), contradicting the fact that $U$ must be a positive quantity. Due to the invariance of the problem under translations, we can assume without lost of generality that $\xi_{0}=0$, modifying the values of $C$ and $K$. Then

$$
U(0)=0, \quad U^{\prime}(0)=0
$$

Imposing these conditions into (4.16), it then follows that:

$$
\begin{aligned}
C & =\frac{2 a\left[1+\mathcal{V}^{2}(1-\Gamma)\right]}{\mathcal{V}\left[\sqrt{\mathcal{V}^{2}+4 \Gamma}-(2 \Gamma-1) \mathcal{V}\right]} \geqslant 0 \\
K & =\frac{a\left(\sqrt{\mathcal{V}^{2}+4 \Gamma}+\mathcal{V}\right)}{(2 \Gamma-1) \mathcal{V}-\sqrt{\mathcal{V}^{2}+4 \Gamma}}
\end{aligned}
$$

Using the formulae (4.15) and (4.17), it follows that

$$
W(0)=\frac{-2 a}{\mathcal{V}\left(\mathcal{V}+\sqrt{\mathcal{V}^{2}+4 \beta}\right)}<0
$$

in contradiction with the fact that $W$ is a positive quantity. This fact does not lead to a contradiction because $\mathcal{V}$ becomes very large as $\varepsilon \rightarrow 0$, therefore in that limit (4.19) implies $W(0)=0$. In any case, we must study in detail the boundary layer that arises as $U$ and $W$ become small.

\subsubsection{Region II $(U \simeq \varepsilon)$ : a key non-linear eigenvalue problem}

Near the point $\xi=0$, the function $U$ behaves quadratically on $\xi$ due to (4.16), (4.18), (4.17), even if $\mathcal{V} \rightarrow \infty$ as $\varepsilon \rightarrow 0$. On the other hand, $W$ is a linear function of $\xi$ near the point $\xi=0$ (see Figure 4.2 ).

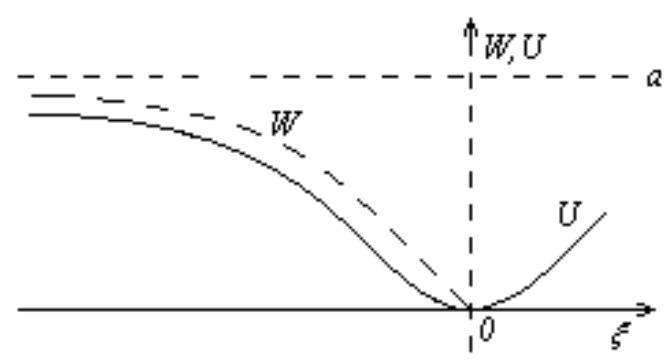

Fig. 4.2: The shape of the functions given in (4.15), (4.16), where the constants $C$ and $K$ are given in (4.17) and (4.18).

Therefore, $U$ rescales as the square of $W$ as $\varepsilon \rightarrow 0$, in the boundary layer in which $U$ becomes of order $\varepsilon$. This provides the rescaling on $\varepsilon$ for $U, W$. In order to obtain the rescalings for $\xi$ and $\mathcal{V}$, we use the rescalings for $U$, $W$ in (4.6)-(4.7) and adjust those of $\xi$ and $\mathcal{V}$ in order to obtain a nontrivial limit problem. This yields to

$$
\xi=\varepsilon^{\frac{1}{4}} \cdot \eta, \quad \mathcal{V}=\varepsilon^{-\frac{1}{4}} \cdot v, \quad U(\xi)=\varepsilon \cdot u(\eta), \quad W(\xi)=\varepsilon^{\frac{1}{2}} \cdot \phi(\eta),
$$

Their substitution into (4.2)-(4.4) gives the system

$$
\begin{aligned}
& -v R_{\eta}(\eta)=\kappa \varepsilon^{\frac{1}{2}}(u+\mathcal{P})\left[\frac{\mu(u+1)-(u+d) R}{\left(u+\frac{1}{c}\right)(u+1)}\right] \\
& -v \phi_{\eta}(\eta)=\frac{b \varepsilon(u+1)^{2}+\Theta R^{2} u^{2}}{(u+1)^{2}+\Lambda R^{2} u^{2}}-\varepsilon^{\frac{1}{2}} \phi \\
& -v u_{\eta}(\eta)=u_{\eta \eta}+\Gamma\left[\phi-\varepsilon^{\frac{1}{2}} u\right]
\end{aligned}
$$


that reduces at the limit $\varepsilon \rightarrow 0$, taking into account Table 4 , to

$$
\begin{aligned}
& -v \phi_{\eta}(\eta)=\frac{\Theta R_{s}^{2} u^{2}}{(u+1)^{2}+\Lambda R_{s}^{2} u^{2}} \\
& -v u_{\eta}(\eta)=u_{\eta \eta}+\Gamma \phi
\end{aligned}
$$

Using the asymptotic behaviour of $U, W$ as $\xi \rightarrow 0$, computed by means of the solution (4.15)-(4.16), as well as (4.20), we obtain the following matching conditions with Region I in the intermediate limit $\xi \rightarrow 0, \eta \rightarrow-\infty$, $\varepsilon \rightarrow 0$ :

$$
\begin{aligned}
& \phi(\eta) \sim-\frac{a}{v} \eta, \quad \text { as } \quad \eta \rightarrow-\infty \\
& u(\eta) \sim \frac{\Gamma a}{2 v^{2}} \eta^{2}, \quad \text { as } \quad \eta \rightarrow-\infty
\end{aligned}
$$

On the other hand, (4.10) yields the following boundary conditions:

$$
\begin{aligned}
& \lim _{\eta \rightarrow \infty} \phi(\eta)=0 \\
& \lim _{\eta \rightarrow \infty} u(\eta)=0
\end{aligned}
$$

Notice that we can simplify the functional dependence of $v$ on the parameters of the system (4.24)-(4.25) by means of the following change of variables:

$$
z=v \eta, \quad w=\frac{v^{2}}{\Theta R_{s}^{2}} \phi, \quad \varkappa=\Lambda R_{s}^{2}, \quad \chi^{-4}=\frac{\Gamma \Theta R_{s}^{2}}{v^{4}}
$$

that transforms (4.24)-(4.29) into the non-linear eigenvalue problem

$$
\begin{aligned}
-w_{z} & =\frac{u^{2}}{(u+1)^{2}+\varkappa u^{2}} \quad-\infty<z<\infty \\
-u_{z} & =u_{z z}+\chi^{-4} w \quad-\infty<z<\infty \\
w(z) & \sim-\frac{1}{1+\varkappa} z, \quad u(z) \sim \frac{\chi^{-4}}{2(1+\varkappa)} z^{2}, \quad z \rightarrow-\infty \\
\lim _{z \rightarrow \infty} w(z) & =0, \quad \lim _{z \rightarrow \infty} u(z)=0
\end{aligned}
$$

We study the problem (4.31)-(4.34) by means of the shooting method in Appendix A, where we show that for each $\varkappa \geqslant 0$ there exists a unique eigenvalue $\chi=\chi(\varkappa)$ solving (4.31)-(4.34). In particular, this non-linear eigenvalue problem provides a method for computing the wave velocity in the considered asymptotic limit. Indeed, taking into account the definition of $\Theta$ (cf. Table 4), (4.30) yields to the functional dependence

$$
v=(\Gamma B \varepsilon)^{1 / 4} \cdot \varkappa^{1 / 4} \cdot \chi(\varkappa)
$$

A plot of the function $\chi(\varkappa)$ can be found in Figure 4.3 .

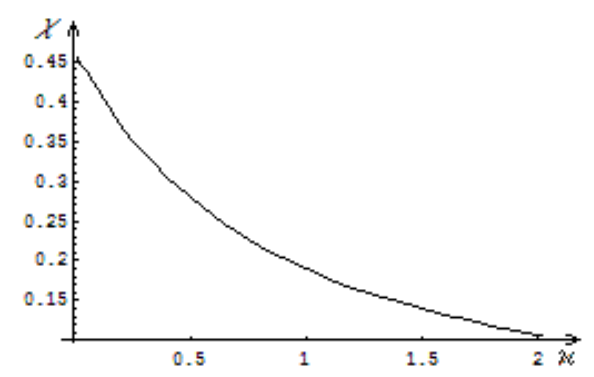

Fig. 4.3: The plot of the function $\chi(\varkappa)$. 


\subsection{Pulse speed. Comparison with experimental data.}

Using (4.35) we can obtain a formula for the pulse speed in the original variables, as a function of the biochemical parameters of the initial problem. Indeed, using (3.6) and (3.24), we obtain that the pulse speed is:

$$
V_{c A M P \text { pulse }}=\frac{d x}{d t}=\sqrt{D\left(k_{i}+k_{t}\right)} \cdot \frac{d \widetilde{x}}{d \tau}
$$

Since the wave speed in the variables $(\widetilde{x}, \tau)$ is $\mathcal{V}$, using formulae $(3.11),(4.20),(4.35)$, and Table 4 , we obtain the following formula for the pulse speed in the considered asymptotic limit:

$$
V_{\text {cAMP pulse }}=\sqrt{D} \cdot\left(\frac{\alpha q \sigma k_{t}}{h(1+\alpha)}\right)^{1 / 4} \cdot \varkappa^{1 / 4} \cdot \chi(\varkappa)
$$

where, taking into account (3.5), (3.16), (3.22), and Table 4, we have

$$
\varkappa=\frac{(1+\alpha) \epsilon}{(1+\alpha \theta)} \cdot\left(\frac{L_{2}\left(c+L_{1}\right)}{1+L_{2}}\right)^{2} \cdot\left(\frac{2 \alpha q \sigma k_{t} \lambda \theta+k_{e} h\left(k_{i}+k_{t}\right)(1+\alpha \theta)}{2 \alpha q \sigma k_{t} \lambda \theta\left(c+L_{1}\right)+k_{e} h\left(k_{i}+k_{t}\right)(1+\alpha \theta)\left(1+L_{1}\right)}\right)^{2}
$$

For the numerical values of the parameters as in Table 3 , we have $\varkappa \approx 0.2966$ and the corresponding eigenvalue $\chi$ of the problem (4.31)-(4.34), numerically computed, is $\chi \approx 0.3377$.

Assuming that the diffusion coefficient for cAMP is $D=4 \times 10^{-6} \mathrm{~cm}^{2} / \mathrm{sec}$ (see, for instance, [19], [20]), the pulse speed would be

$$
V_{\text {pulse }} \approx 162 \mu \mathrm{m} / \mathrm{min}
$$

which agrees with the order of magnitude obtained in the experimental observations (see, for example, [10], [14], [19], [20]).

\subsection{The top of the pulse (region of high concentration of $W$ and $U$ )}

The analysis in the Section 4.1 was made in the assumption that $R$ remains close to $R_{s}$ in the leading front. Moreover, for $\xi \rightarrow-\infty, U$ and $W$ approach in a shorter time scale their equilibrium value of the excited state given in (4.8). So, it does not provide a uniformly valid approximation for negative large values of $\xi$. This means that in a longer time scale, after the front, we cannot assume that the $R$-component of the solution of the system (4.2)-(4.4) remains constant.

Indeed, we readily check that $R$ becomes of order one at distances $\xi$ of order $\kappa^{-1} \varepsilon^{-1 / 4}$. This suggests the introduction of a new rescaling as follows

$$
\widetilde{\xi}=\frac{\kappa \varepsilon^{1 / 4}}{v} \cdot \xi
$$

Taking also into account that $\mathcal{V}=\varepsilon^{-\frac{1}{4}} \cdot v(\operatorname{cf} .(4.20))$, the equations (4.2)-(4.4) become

$$
\begin{aligned}
-R_{\widetilde{\xi}}(\widetilde{\xi}) & =(U+\mathcal{P} \varepsilon)\left[\frac{\mu(U+\varepsilon)-(U+d \varepsilon) R}{\left(U+\frac{\varepsilon}{c}\right)(U+\varepsilon)}\right] \\
-\kappa W_{\widetilde{\xi}}(\widetilde{\xi}) & =\frac{b \varepsilon(U+\varepsilon)^{2}+\Theta R^{2} U^{2}}{(U+\varepsilon)^{2}+\Lambda R^{2} U^{2}}-W \\
-\kappa U_{\widetilde{\xi}}(\widetilde{\xi}) & =\kappa^{2} \varepsilon^{1 / 2} U_{\widetilde{\xi} \widetilde{\xi}}+\Gamma[W-U]
\end{aligned}
$$

which, using Table 4, reduce in the considered asymptotic limit to

$$
\begin{aligned}
R_{\widetilde{\xi}}(\widetilde{\xi}) & =\frac{R}{U} \cdot(U+\mathcal{P} \varepsilon) \\
W(\widetilde{\xi}) & =U(\widetilde{\xi})=\frac{\Theta R^{2}}{1+\Lambda R^{2}}
\end{aligned}
$$

The matching with the leading front requires

$$
\begin{aligned}
R(0) & =R_{s} \\
\lim _{\widetilde{\xi} \rightarrow 0} U(\widetilde{\xi}) & =\lim _{\xi \rightarrow-\infty} U(\xi)=a \\
\lim _{\widetilde{\xi} \rightarrow 0} W(\widetilde{\xi}) & =\lim _{\xi \rightarrow-\infty} W(\xi)=a
\end{aligned}
$$


Then, in this part of the front, $R$ satisfies the following equation:

$$
R_{\widetilde{\xi}}(\widetilde{\xi})=\frac{1}{\Theta R}\left[\mathcal{P} \varepsilon+(\Theta+\Lambda \mathcal{P} \varepsilon) R^{2}\right]
$$

The solution of (4.46), that satisfies the condition (4.43), is given by

$$
R(\widetilde{\xi})=\sqrt{\frac{\mathcal{P} \varepsilon}{\Theta+\Lambda \mathcal{P} \varepsilon}\left[e^{\frac{2(\Theta+\Lambda \mathcal{P} \varepsilon)}{\Theta}\left(\widetilde{\xi}-\widetilde{\xi}_{1}\right)}-1\right]}
$$

where

$$
\widetilde{\xi}_{1}=\frac{\Theta}{2(\Theta+\Lambda \mathcal{P} \varepsilon)} \ln \frac{\mathcal{P} \varepsilon}{\mathcal{P} \varepsilon+R_{s}^{2}(\Theta+\Lambda \mathcal{P} \varepsilon)}<0
$$

Using (4.42), we obtain

$$
U(\widetilde{\xi})=W(\widetilde{\xi})=\frac{\Theta}{\Lambda} \cdot \frac{e^{\frac{2(\Theta+\Lambda \mathcal{P} \varepsilon)}{\Theta}\left(\widetilde{\xi}-\widetilde{\xi}_{1}\right)}-1}{e^{\frac{2(\Theta+\Lambda \mathcal{P} \varepsilon)}{\Theta}\left(\widetilde{\xi}-\widetilde{\xi}_{1}\right)+\frac{\Theta}{\Lambda \mathcal{P} \varepsilon}}}
$$

The approximation formulae (4.47) and (4.49) are valid in the interval $\widetilde{\xi}_{1} \leqslant \widetilde{\xi} \leqslant 0$. The shape of the solutions in this region is given in Figure 4.4.

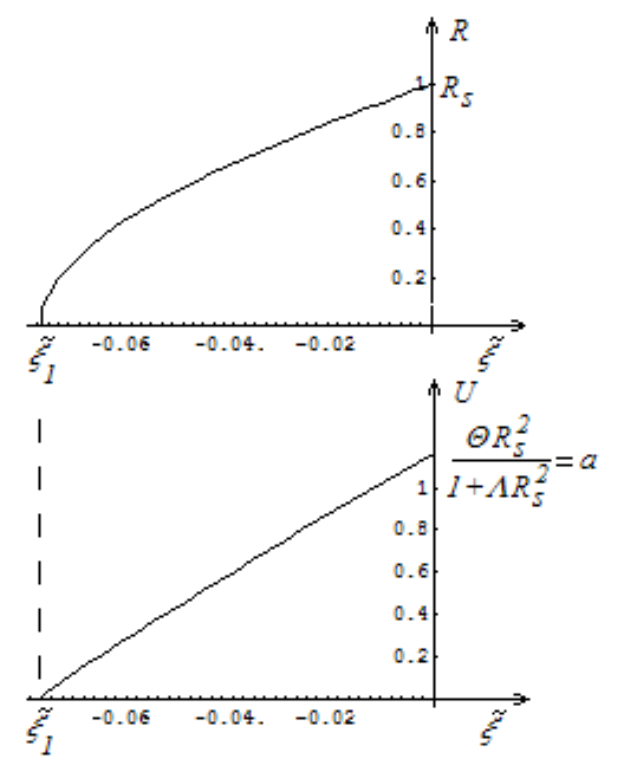

Fig. 4.4: The shape of the solutions $R$ and $U$ of the system (4.2)-(4.4) in the region of high concentration (the curves are obtained for the numerical values of the parameters as in Table 3$)$.

In the initial coordinates $(x, t)$, the time that a given cell remains in the excited state and, respectively, the width of the pulse, as functions of the initial biochemical parameters, are:

$$
\begin{gathered}
t_{\text {exc }}=\frac{1}{2 k_{2}\left(1+L_{2}\right)}\left(1-\frac{1}{\Upsilon}\right) \ln (1+\Upsilon \varkappa) \\
x_{\text {width }}=\frac{\sqrt{D}}{2 k_{2}\left(1+L_{2}\right)} \cdot\left(\frac{\alpha q \sigma k_{t}}{h(1+\alpha)}\right)^{1 / 4} \cdot\left(1-\frac{1}{\Upsilon}\right) \cdot \varkappa^{1 / 4} \cdot \chi(\varkappa) \cdot \ln (1+\Upsilon \varkappa)
\end{gathered}
$$

where

$$
\Upsilon=1+\frac{\alpha q \sigma k_{t} k_{2}}{k_{1} k_{e} h\left(k_{i}+k_{t}\right)(1+\alpha)}
$$

and $\varkappa$ is given in $(4.37)$.

Let us remark that, for the numerical values of the parameters as in Table 3 and taking into account (4.51), we have $x_{w i d t h}=1848.5 \mu \mathrm{m}$. This value is consistent with the experimental observations (see, for example, [10]). 


\subsection{The trailing front}

Although the description of the pulse given in Sections 4.1 and 4.3 provides formulae for the concentrations in the most relevant regions of the pulse, we will derive in the rest of this section an asymptotic description of the wave in the trailing front, where the concentrations are close to equilibrium. The values of $U$ and $W$ are very close to their equilibrium value, and therefore the effect of this boundary layer in the shape of the pulse is very small. However, we shall study this region in detail for mathematical self-consistency, in order to verify that the sought-for pulse exist.

The main reason for the arguments in the rest of this Section is analogous to the one used in the classical analysis of the FHN model, namely for small enough values of $R$, the branch of excited equilibrium states ceases to exist, therefore the values of the concentrations return to the equilibrium value. Nevertheless, there are several technical differences due to the fact that the model considered is three-dimensional instead of twodimensional, and the rescaling of the parameters is different from the usual one in the analysis of the FHN model.

In the analysis in the Section 4.3 it has been assumed that $W$ and $U$ are of order one. In the region where $U$ becomes again of order $\varepsilon$, the analysis becomes different as it happened in the analysis of the leading front. We will distinguish several regions depending on the magnitude of the concentrations.

\subsubsection{Region $\mathrm{I}(U \simeq \varepsilon)$}

In order to study the last part of the pulse, we need to introduce a further layer, where a downjump for the variable $U$ will occur. In the considered asymptotic limit, the problem (4.2)-(4.4) has a second boundary layer at $\widetilde{\xi}=\widetilde{\xi}_{1}$ (at which $U$ vanishes). Because the thickness of the boundary layer is of order $\kappa$, the convenient rescaling is the following:

$$
\widetilde{\eta}=\kappa^{-1} \cdot\left(\widetilde{\xi}-\widetilde{\xi}_{1}\right), \quad R(\widetilde{\xi})=\varepsilon^{\frac{1}{2}} \cdot \widetilde{r}(\widetilde{\eta}), \quad W(\widetilde{\xi})=\varepsilon \cdot \widetilde{w}(\widetilde{\eta}), \quad U(\widetilde{\xi})=\varepsilon \cdot \widetilde{u}(\widetilde{\eta})
$$

Note that the speed of the leading front of the pulse is the same for the trailing front. Letting $\varepsilon \rightarrow 0$ and taking into account (3.28), (3.29), (3.30), (4.52), and Table 4, the system (4.38)-(4.40) can be approximated in this region by:

$$
\begin{aligned}
& \widetilde{r}_{\widetilde{\eta}}=\kappa \mathcal{P}\left[\frac{\widetilde{r}}{\widetilde{u}+1}\right] \\
& \widetilde{w}_{\widetilde{\eta}}=\widetilde{w}-\Theta \frac{\widetilde{r}^{2} \widetilde{u}^{2}}{(\widetilde{u}+1)^{2}} \\
& \widetilde{u}_{\widetilde{\eta}}=\Gamma[\widetilde{u}-\widetilde{w}]
\end{aligned}
$$

The outer solutions $(R(\widetilde{\xi}), W(\widetilde{\xi}), U(\widetilde{\xi}))$ and the inner solutions $(\widetilde{r}(\widetilde{\eta}), \widetilde{w}(\widetilde{\eta}), \widetilde{u}(\widetilde{\eta}))$ must match asymptotically. In the considered asymptotic limit, this asymptotic match, between the top and the trailing front of the pulse, occurs on the overlap region that is defined by the intermediate limit $\widetilde{\xi} \rightarrow \widetilde{\xi}_{1}, \widetilde{\eta} \rightarrow \infty, \varepsilon \rightarrow 0$. Taking into account (4.47), (4.49) and (4.52), we obtain the matching conditions:

$$
\begin{aligned}
& \widetilde{r}(\widetilde{\eta}) \sim \sqrt{\frac{\kappa \mathcal{P}}{\Theta} \widetilde{\eta}} \quad \text { as } \quad \widetilde{\eta} \rightarrow+\infty \\
& \widetilde{w}(\widetilde{\eta}) \sim \kappa \mathcal{P} \widetilde{\eta} \quad \text { as } \quad \tilde{\eta} \rightarrow+\infty \\
& \widetilde{u}(\widetilde{\eta}) \sim \kappa \mathcal{P} \widetilde{\eta} \quad \text { as } \quad \tilde{\eta} \rightarrow+\infty
\end{aligned}
$$

Standard computations yield that the asymptotics of the solutions of (4.53)-(4.58) are

$$
\begin{aligned}
& \widetilde{r}(\widetilde{\eta}) \sim C_{1} e^{\kappa \mathcal{P} \widetilde{\eta}} \quad \text { as } \quad \widetilde{\eta} \rightarrow-\infty \\
& \widetilde{w}(\widetilde{\eta}) \sim C_{2} e^{\tilde{\eta}}+C_{4} e^{2(\kappa \mathcal{P}+\Gamma) \tilde{\eta}} \quad \text { as } \quad \widetilde{\eta} \rightarrow-\infty \\
& \widetilde{u}(\widetilde{\eta}) \sim C_{3} e^{\widetilde{\eta}}+C_{5} e^{\Gamma \widetilde{\eta}} \quad \text { as } \quad \widetilde{\eta} \rightarrow-\infty
\end{aligned}
$$

for suitable positive constants of order one, $C_{i}, i=1, \ldots, 5$. Notice, in particular, that $C_{3}=\frac{\Gamma C_{2}}{\Gamma-1}$.

Multiple cases arise depending on the relative size of the coefficients of the exponential factors in these formulae. However, we will consider the case of the parameters with numerical values as in Table 3 and we shall consider $\Gamma>1$, since the other cases can be treated with similar methods. In this case, a plot of the functions $\widetilde{r}$ and $\widetilde{u}$ is given in Figure 4.5. 


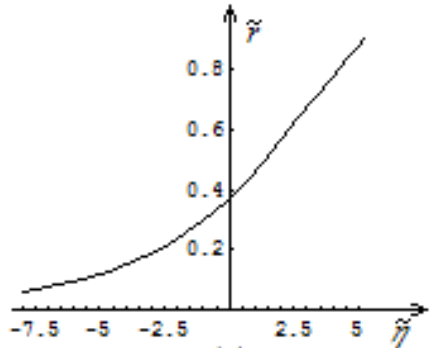

(a)

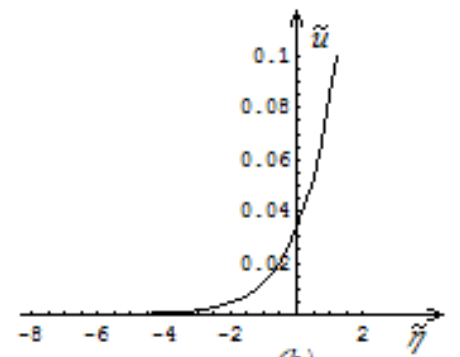

(b)

Fig. 4.5: The $\widetilde{r}$-component $(a)$ and $\widetilde{u}$-component $(b)$ of the solution of the system (4.53)-(4.55), obtained by numerical integration. Parameter values are taken from Table 3.

\subsubsection{Region II $(U R \simeq \mu \varepsilon)$}

The previous analysis is valid only for $U R \gg \mu \varepsilon$. To deal with the region where $U R \simeq \mu \varepsilon, R \ll \varepsilon^{\frac{1}{2}}, d \varepsilon \ll U \ll \varepsilon$, it is convenient to introduce a new set of variables, by setting:

$$
\zeta=\widetilde{\eta}-\widetilde{\eta}_{\varepsilon}, \quad \widetilde{r}(\widetilde{\eta})=\left(\frac{\mu}{\sqrt{\varepsilon}}\right)^{\frac{\kappa \mathcal{P}}{\kappa \mathcal{P}+1}} \cdot r(\zeta), \quad \widetilde{w}(\widetilde{\eta})=\left(\frac{\mu}{\sqrt{\varepsilon}}\right)^{\frac{1}{\kappa \mathcal{P}+1}} \cdot w(\zeta), \quad \widetilde{u}(\widetilde{\eta})=\left(\frac{\mu}{\sqrt{\varepsilon}}\right)^{\frac{1}{\kappa \mathcal{P}+1}} \cdot u(\zeta)
$$

where $\widetilde{\eta}_{\varepsilon}=\ln \left(\frac{\mu}{\sqrt{\varepsilon}}\right)^{\frac{1}{\kappa \mathcal{P}+1}}$. Using this change of variables and taking into account $(3.31),(3.32)$, (4.52), and Table 4, in the considered asymptotic limit the problem (4.38)-(4.40) can be approximated by:

$$
\begin{aligned}
r_{\zeta} & =\kappa \mathcal{P}\left[r-\frac{1}{u}\right] \\
w_{\zeta} & =w \\
u_{\zeta} & =\Gamma[u-w]
\end{aligned}
$$

The solutions of this problem must satisfy the matching conditions with $(\widetilde{r}(\widetilde{\eta}), \widetilde{w}(\widetilde{\eta}), \widetilde{u}(\widetilde{\eta}))$ in the intermediate scale $\zeta \rightarrow+\infty, \widetilde{\eta} \rightarrow-\infty, \varepsilon \rightarrow 0$. So, from (4.63), (4.64) and taking into account (4.59)-(4.61), we see that

$$
w(\zeta)=C_{2} e^{\zeta}, \quad u(\zeta)=C_{3} e^{\zeta}+C_{5} e^{\Gamma \zeta}
$$

Let us remark that the asymptotics of the $r$-component of the solution of the system (4.62)-(4.64) are

$$
\begin{aligned}
r(\zeta) & \sim C_{1} e^{\kappa \mathcal{P} \zeta} \quad \text { as } \quad \zeta \rightarrow+\infty \\
r(\zeta) & \sim C_{6} e^{-\zeta} \quad \text { as } \quad \zeta \rightarrow-\infty
\end{aligned}
$$

where $C_{6}=\frac{\kappa \mathcal{P}}{C_{3}(\kappa \mathcal{P}+1)}$ is a positive constant. Representations of the functions $r$ and $u$ are given in Figure 4.6.

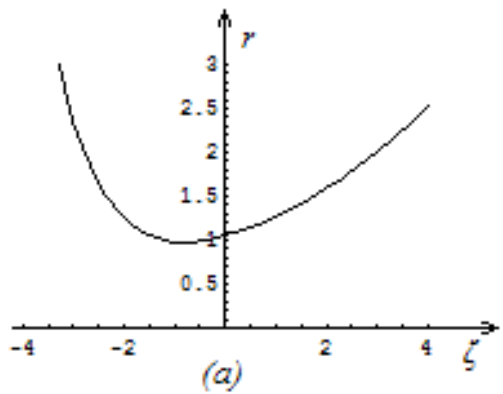

(a)

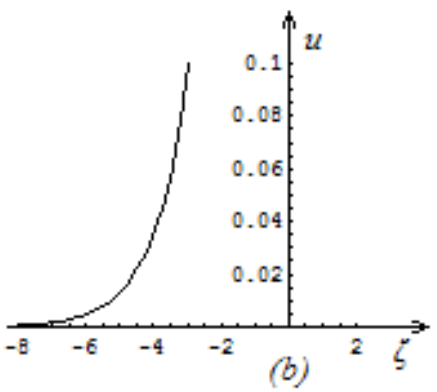

(b)

Fig. 4.6: The $r$-component $(a)$ and $u$-component $(b)$ of the solution of the system(4.62)-(4.64), obtained by numerical integration. The parameter values are the same as in Table 3. 
Therefore, matching $u(\zeta)$ and $\widetilde{u}(\widetilde{\eta})$ still does not provide a valid representation for the pulse and we need to introduce a further layer, near the value $\zeta_{\varepsilon}=\ln \left(\left(\frac{\mu}{\sqrt{\varepsilon}}\right)^{\frac{\kappa \mathcal{P}}{\kappa \mathcal{P}+1}}\right)$.

\subsubsection{Region III}

The analysis for the previous region is no longer valid when $U \simeq d \varepsilon$. In this case some of the terms we neglected previously become essential and we must take them into account. To deal with the corresponding region, it is convenient to introduce the rescaling:

$$
\begin{gathered}
\widetilde{\zeta}=\zeta-\zeta_{\varepsilon}, \quad r(\zeta, \varepsilon)=\frac{1}{d}\left(\frac{\mu}{\sqrt{\varepsilon}}\right)^{\frac{1}{\kappa \mathcal{P}+1}} \widetilde{R}(\widetilde{\zeta}, \varepsilon), \\
w(\zeta, \varepsilon)=d\left(\frac{\mu}{\sqrt{\varepsilon}}\right)^{-\frac{1}{\kappa \mathcal{P}+1}} \widetilde{W}(\widetilde{\zeta}, \varepsilon), \quad u(\zeta, \varepsilon)=d\left(\frac{\mu}{\sqrt{\varepsilon}}\right)^{-\frac{1}{\kappa \mathcal{P}+1}} \widetilde{U}(\widetilde{\zeta}, \varepsilon)
\end{gathered}
$$

Letting $\varepsilon \rightarrow 0$ and taking into account the previous changes of the variables, (3.19), (3.31), and Table 4, the problem (4.38)-(4.40) can be approximated by:

$$
\begin{aligned}
\widetilde{R}_{\widetilde{\zeta}} & =\kappa \mathcal{P}\left[\frac{(\widetilde{U}+1) \widetilde{R}-1}{\widetilde{U}}\right] \\
\widetilde{W}_{\widetilde{\zeta}} & =\widetilde{W}-\frac{\Theta \mu^{2}}{d \varepsilon} \widetilde{R}^{2} \widetilde{U}^{2} \\
\widetilde{U}_{\widetilde{\zeta}} & =\Gamma[\widetilde{U}-\widetilde{W}]
\end{aligned}
$$

To allow the matching with Region II in the intermediate scale $\widetilde{\zeta} \rightarrow+\infty, \zeta \rightarrow-\infty, \varepsilon \rightarrow 0$, we also require

$$
\begin{aligned}
\widetilde{R}(\widetilde{\zeta}) & \sim C_{6} e^{-\widetilde{\zeta}} & & \text { as } & \widetilde{\zeta} \rightarrow+\infty \\
\widetilde{W}(\widetilde{\zeta}) & \sim C_{2} e^{\widetilde{\zeta}} & & \text { as } & \widetilde{\zeta} \rightarrow+\infty \\
\widetilde{U}(\zeta) & \sim C_{3} e^{\widetilde{\zeta}}+C_{5} e^{\Gamma \zeta} & & \text { as } & \widetilde{\zeta} \rightarrow+\infty
\end{aligned}
$$

We have solved the problem (4.65)-(4.70) numerically for the numerical value of the parameters as in Table 3. We have obtained that the corresponding solution $\widetilde{R}$ converges to $\widetilde{R}=1$, as $\widetilde{\zeta} \rightarrow-\infty$. A plot of the function $\widetilde{R}$ is given in Figure 4.7.
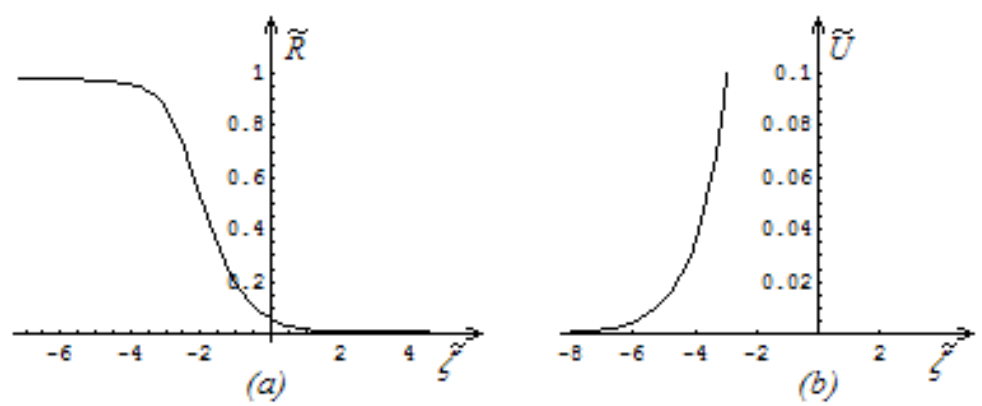

Fig. 4.7: The $\widetilde{R}$-component $(a)$ and $\widetilde{U}$-component $(b)$ of the solution of the system (4.65)-( 4.67), obtained by numerical integration. Parameter values are taken from Table 3.

As a consequence, the asymptotics of $\widetilde{W}$ and $\widetilde{U}$ are given by

$$
\begin{aligned}
\widetilde{W}(\widetilde{\zeta}) & \sim C_{2} e^{\widetilde{\zeta}} & & \text { as } \quad \widetilde{\zeta} \rightarrow-\infty \\
\widetilde{U}(\widetilde{\zeta}) & \sim C_{3} e^{\widetilde{\zeta}}+C_{5} e^{\Gamma \widetilde{\zeta}} & & \text { as } \quad \widetilde{\zeta} \rightarrow-\infty
\end{aligned}
$$




\subsubsection{Region IV}

To complete the picture of the trailing front of the pulse, a further region is needed, namely the region where $b \varepsilon \ll U \ll d \varepsilon$. In this region $R$ stabilizes to the equilibrium value $\frac{\mu}{d} \sim 1$ (cf. (3.29)). It is convenient to introduce the rescaling:

$$
\bar{\varrho}=\widetilde{\zeta}-\widetilde{\zeta}_{\varepsilon}, \quad \widetilde{W}(\widetilde{\zeta} ; \varepsilon)=\frac{\sqrt{b \varepsilon}}{\mu} \cdot \bar{W}(\bar{\varrho} ; \varepsilon), \quad \widetilde{U}(\widetilde{\zeta} ; \varepsilon)=\frac{\sqrt{b \varepsilon}}{\mu} \cdot \bar{U}(\bar{\varrho} ; \varepsilon)
$$

where $\widetilde{\zeta}_{\varepsilon}=\ln \left(\frac{\sqrt{b \varepsilon}}{\mu}\right)$.

Using this change of the variables and the previous ones, and taking into account (3.31), (3.32) and Table 4, the problem (4.38)-(4.40) can be approximated by :

$$
\begin{aligned}
\bar{W}_{\bar{\varrho}} & =\bar{W} \\
\bar{U}_{\bar{\varrho}} & =\Gamma[\bar{U}-\bar{W}]
\end{aligned}
$$

The solutions of this system, taking into account (4.71), (4.72), are

$$
\bar{W}(\bar{\varrho})=C_{2} e^{\bar{\varrho}}, \quad \bar{U}(\bar{\varrho})=C_{3} e^{\bar{\varrho}}+C_{5} e^{\Gamma \bar{\varrho}}
$$

\subsubsection{Region V}

This region, where $U \sim b \varepsilon$, concludes the shape of the pulse. To deal with it, it is convenient to introduce the rescaling:

where $\bar{\varrho}_{\varepsilon}=\ln (b \varepsilon)$.

$$
\varrho=\bar{\varrho}-\bar{\varrho}_{\varepsilon}, \quad \bar{W}(\varrho ; \varepsilon)=\frac{b \mu}{d \sqrt{b \varepsilon}} \cdot \mathcal{W}(\varrho ; \varepsilon), \quad \bar{U}(\bar{\varrho} ; \varepsilon)=\frac{b \mu}{d \sqrt{b \varepsilon}} \cdot \mathcal{U}(\varrho ; \varepsilon)
$$

Using these variables and taking into account (3.31), (3.32) and Table 4, the problem (4.38)-(4.40) reduces in the considered asymptotic limit to :

$$
\begin{aligned}
& \mathcal{W}_{\varrho}=\mathcal{W}-1 \\
& \mathcal{U}_{\varrho}=\Gamma[\mathcal{U}-\mathcal{W}]
\end{aligned}
$$

It is easy to see that the corresponding solutions $\mathcal{W}$ and $\mathcal{U}$ stabilize to $\mathcal{W}=1$ and $\mathcal{U}=1$, as $\varrho \rightarrow-\infty$. In this way, the trailing front for the $U$-component of the pulse has been sketched.

\section{Conclusions}

In this paper we presented two continuum models for cAMP relay response in cellular slime molds Dictyostelium discoideum, based on the kinetics of the MG model and, respectively, GS model. For both models we have considered the one-dimensional spatial problem and we have made the assumption that extracellular cAMP is the only diffusible chemical of the system.

We have shown mathematically that, for these reaction-diffusion models, we can obtain pulse-like solution using singular perturbation methods. In particular, we have obtained detailed formulae for the wave speed and for the concentration profiles in suitable limits. We have obtained that the wave speed is determined in this regime by means of the solutions of a non-linear eigenvalue problem.

The main results that we have obtained with our analysis are formulae for the wave speed of the pulse (4.36), for the width of the pulse (4.51), and for the time that the cells remain in the excited state (4.50).

Most likely, similar mathematical methods can be used to study other available models of wave propagation in the literature as, for instance, the Tang-Othmer model [22].

As a final remark, we draw attention to the fact that the numerical values obtained in the analysis of the MG model agree with experimental results.

\section{Acknowledgements}

The authors would like to thank Marek Bodnar and Miguel A. Herrero for several valuable discussions and for their interesting remarks. This work was partially supported by the Research Training Network "Using Mathematical Modelling and Computer Simulation to Improve Cancer Therapy" (HPRN-CT-2000-00105) and by DGES Grant MTM2004-05634. 


\section{APPENDIX A: Asymptotic notation. Analysis of the nonlinear eigenvalue problems}

We begin by recalling the symbols of the asymptotic order relations that express the relative magnitudes of two functions.

Definition 7.1 (asymptotic order relations)

Let $f$ and $g$ two functions defined in a neighbourhood of $x_{0}$, that do not vanish on their domain.

- The notation

$$
f(x) \ll g(x), \quad x \rightarrow x_{0}
$$

which is read " $f(x)$ is much smaller than $g(x)$ as $x$ tends to $x_{0} "$, means $\lim _{x \rightarrow x_{0}}[f(x) / g(x)]=0$.

- The notation

$$
f(x) \sim g(x), \quad x \rightarrow x_{0}
$$

which is read " $f(x)$ is asymptotic to $g(x)$ as $x$ tends to $x_{0} "$, means $\lim _{x \rightarrow x_{0}}[f(x) / g(x)]=1$, or, equivalently, $f(x)-g(x) \ll g(x)$ as $x \rightarrow x_{0}$.

- The notation

$$
f(x)=\mathcal{O}[g(x)], \quad x \rightarrow x_{0}
$$

which is read " $f(x)$ is at most of order $g(x)$ as $x$ tends to $x_{0} "$, means $|f(x) / g(x)|<M$, for some constant $M$ if $x$ is in a neighbourhood of $x_{0}$.

Remark 7.1 We have used also the notation $f(x) \simeq g(x), x \rightarrow x_{0}$ to indicate in an informal, non-rigorous manner that the functions $f$ and $g$ have the same order of magnitude.

In the remainder of this Appendix we study the nonlinear eigenvalue problem (4.31)-(4.34), that we recall here for the reader's convenience:

$$
\begin{aligned}
-w_{z} & =\frac{u^{2}}{(u+1)^{2}+\varkappa u^{2}} \quad-\infty<z<\infty \\
-u_{z} & =u_{z z}+\chi^{-4} w \quad-\infty<z<\infty \\
w & \sim-\frac{1}{1+\varkappa} z, \quad u \sim \frac{\chi^{-4}}{2(1+\varkappa)} z^{2}, \quad z \rightarrow-\infty \\
\lim _{z \rightarrow+\infty} w & =0, \quad \lim _{z \rightarrow+\infty} u=0
\end{aligned}
$$

Theorem 7.1 For each $\varkappa \geqslant 0$ there exists at least a value of $\chi>0$ such that there is a solution of (7.1)-(7.4). Proof. We use a standard "shooting" method. For each fixed $\varkappa$ and $\chi$ there exists a unique positive solution of the system (7.1)-(7.2) satisfying the limit condition (7.4). Such a solution has the following asymptotic behaviour

$$
u(z) \sim C e^{-z}, \quad w(z) \sim \frac{C^{2}}{2} e^{-2 z}, \quad z \rightarrow+\infty
$$

Moreover, there exists a unique positive solution of (7.1)-(7.2) with the asymptotics (7.5) for each value of the positive constant $C$ and since the problem (7.1)-(7.4) is invariant under translations, we can assume without lost of generality that $C=1$. Integrating the equation (7.2) we obtain:

$$
u_{z}(z)=e^{-z}\left(\chi^{-4} \int_{z}^{\infty} w(\tau) e^{\tau} d \tau-1\right)
$$

The asymptotics of $u_{z}$ is given by $u_{z}(z) \sim L(\chi) e^{-z}$ as $z \rightarrow-\infty$, where

$$
L(\chi)=\chi^{-4} \int_{-\infty}^{\infty} w(\tau) e^{\tau} d \tau-1
$$

Due to (7.1), $W$ is bounded linearly as $z \rightarrow-\infty$. Then, the integral in (7.7) converges and it is not hard to see that $L(\chi)$ is a continuous function of $\chi$. Therefore, in order to prove the theorem, we only need to check that $L(\chi)$ has different signs as $\chi \rightarrow+\infty, \chi \rightarrow 0^{+}$. 


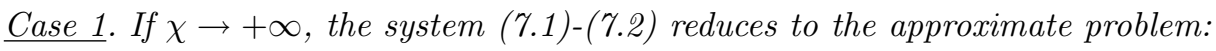

$$
\begin{array}{rlrl}
-w_{z} & =\frac{u^{2}}{(u+1)^{2}+\varkappa u^{2}} & -\infty<z<\infty \\
-u_{z}=u_{z z} & -\infty<z<\infty
\end{array}
$$

Taking into account the boundary conditions (7.5), with $C=1$, the solution of this problem is given by:

$$
\begin{aligned}
u(z) & =e^{-z} \\
w(z) & =\int_{z}^{\infty} \frac{e^{-2 t}}{\left(1+e^{-t}\right)^{2}+\varkappa e^{-2 t}} d t
\end{aligned}
$$

Notice that

$$
\begin{aligned}
& w(z) \sim-\frac{1}{1+\varkappa} z, \quad z \rightarrow-\infty \\
& w(z) \sim \frac{1}{2} e^{-2 z}, \quad z \rightarrow \infty
\end{aligned}
$$

Therefore, $L(\chi)$ defined in (7.7) satisfies:

$$
L(\chi) \rightarrow-1, \quad \chi \rightarrow \infty
$$

Case 2. We now study the limit case $\chi \rightarrow 0^{+}$. To this end, we introduce the new variables:

$$
\widetilde{u}=\chi^{-4} u, \quad \widetilde{w}=\chi^{-8} w, \quad \widetilde{z}=z+\log \left(\chi^{4}\right)
$$

Therefore, in the limit $\chi \rightarrow 0^{+}$(7.1), (7.2) reduce to the system

$$
\begin{aligned}
-\widetilde{w}_{\widetilde{z}} & =\widetilde{u}^{2} \\
-\widetilde{u}_{\widetilde{z}} & =\widetilde{u}_{z z}+\widetilde{w}
\end{aligned}
$$

together with the boundary conditions

$$
\begin{array}{ll}
\widetilde{w}(\widetilde{z}) \sim \frac{1}{2} e^{-2 \widetilde{z}}, & \widetilde{z} \rightarrow \infty \\
\widetilde{u}(\widetilde{z}) \sim e^{-\widetilde{z}}, & \widetilde{z} \rightarrow \infty
\end{array}
$$

Integrating the equation (7.10), it follows that

$$
\widetilde{u}_{\widetilde{z}}(\widetilde{z})=e^{-\widetilde{z}}\left(\int_{\widetilde{z}}^{\infty} \widetilde{w}(\tau) e^{\tau} d \tau-1\right)
$$

We notice that, from (7.9) and (7.11), we deduce that $\widetilde{w}(\widetilde{z})$ decreases as $\widetilde{z}$ increases, but remains positive for all $\widetilde{z} \in \mathbb{R}$.

Let us define

$$
I=\lim _{\tilde{z} \rightarrow-\infty}\left(\int_{\widetilde{z}}^{\infty} \widetilde{w}(\tau) e^{\tau} d \tau-1\right)
$$

As $\widetilde{w}(\tau) e^{\tau}>0$ for all $\tau \in \mathbb{R}$, this limit exists and it is positive (perhaps $\left.+\infty\right)$. Then $I \in(-1,+\infty]$. Let us distinguish now several cases:

(i) Suppose that $I<0$. Then (7.13) implies $\widetilde{u} \sim I e^{-\widetilde{z}}$ as $\widetilde{z} \rightarrow-\infty$. Moreover, from (7.9) we have $\widetilde{w} \sim \frac{I^{2}}{2} e^{-2 \widetilde{z}}$ as $\widetilde{z} \rightarrow-\infty$. However, this would imply that $\lim _{\widetilde{z} \rightarrow-\infty}\left(\int_{\widetilde{z}}^{\infty} \widetilde{w}(\tau) e^{\tau} d \tau-1\right)>0$, and this yields to a contradiction. Henceforth $I \geqslant 0$. 
(ii) Suppose now that $I=0$. It then follows that

$$
\widetilde{u}_{\widetilde{z}}(\widetilde{z})=-e^{-\widetilde{z}} \int_{-\infty}^{\widetilde{z}} \widetilde{w}(\tau) e^{\tau} d \tau<0
$$

Since $\widetilde{w}$ is a decreasing positive function, we get from the last relation that $\widetilde{u}_{\widetilde{z}}(\widetilde{z}) \leqslant-\widetilde{w}(\widetilde{z})$. Differentiating in (7.9), we obtain $\widetilde{w}_{\tilde{z} \widetilde{z}}=-2 \widetilde{u} \widetilde{u}_{\widetilde{z}}$ and then $\widetilde{w}_{\tilde{z} \tilde{z}} \geqslant 2 \widetilde{u} \widetilde{w}$.

From (7.12) and (7.15) we deduce that $\widetilde{u}(\widetilde{z})$ decreases as $\widetilde{z}$ increases and remains positive for all $\widetilde{z} \in \mathbb{R}$. Let $C>1$ and $\widetilde{z}_{0} \in \mathbb{R}$ fixed such that $2 u(\widetilde{z})>C$, for all $\widetilde{z}<\widetilde{z}_{0}$. Then $\widetilde{w}_{\tilde{z} \widetilde{z}}(\widetilde{z}) \geqslant C \widetilde{w}(\widetilde{z})$, for all $\widetilde{z}<\widetilde{z}_{0}$, which implies that $\widetilde{w}(\widetilde{z}) \geqslant \widetilde{w}\left(\widetilde{z}_{0}\right) e^{\sqrt{C}\left(\widetilde{z}-\widetilde{z}_{0}\right)}$, for all $\widetilde{z}<\widetilde{z}_{0}$. This argument and (7.11) lead to $I=+\infty$, hence to a contradiction. Therefore, the only possible case is $I>0$.

(iii) We have then obtained that $I>0$. Using (7.8) and (7.14), we obtain

$$
L(\chi)=\chi^{-4} \int_{-\infty}^{\infty} w(\tau) e^{\tau} d \tau-1=\int_{-\infty}^{\infty} \widetilde{w}(\tau) e^{\tau} d \tau-1>0
$$

Summarizing, $L(\chi)$ changes the sign as $\chi$ varies from 0 to $+\infty$. In the limit $\chi \rightarrow+\infty$, the function $u$ increases exponentially as $z$ decreases to $-\infty$ and in the limit $\chi \rightarrow 0^{+}$, the function $u$ becomes negative at some finite value of $z$. We illustrate the idea of the proof in Figure \%.1.

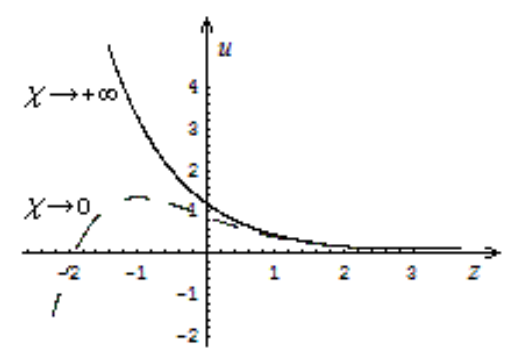

Fig. 7.1: The curves represent the numeric $u$-approximation for the problem (4.39)-( 4.40) (solid line) and respectively the numeric $\widetilde{u}$-approximation for the problem (7.9)-(7.10) (dashed line) using the parameters from Table III. The representation scales of the two curves are not the same. The dashed curve is the representation of $\widetilde{u}=\frac{\Gamma \Theta R_{s}^{2}}{v^{4}} u$ for $v \rightarrow 0$ (by respect to $\widetilde{z}=z+\log \left(v^{4}\right)$ ), so on the same scale it would be much "smaller".

As $L(\chi)$ is a continuous function and changes the sign as $\chi$ varies from 0 to $+\infty$, there exists $\chi_{0}>0$ such that $L\left(\chi_{0}\right)=0$. Then (7.6) implies

$$
u_{z}(z)=-\chi_{0}^{-4} e^{-z} \int_{-\infty}^{z} w(\tau) e^{\tau} d \tau<0
$$

This means that the function $u(z)$ decreases as $z$ increases and then the limit $\lim _{z \rightarrow-\infty} u(z)$ exists, perhaps infinity. Let us write

$$
\lim _{z \rightarrow-\infty} u(z)=l \in(0, \infty]
$$

From (7.1) we obtain

$$
D_{1}=\lim _{z \rightarrow-\infty} w_{z}=-\frac{l^{2}}{(l+1)^{2}+\varkappa l^{2}}, \quad l \in(0, \infty]
$$

where the right side of the second equality is considered in the sense of limit if $l=\infty$. Then $w(z) \sim D_{1} z$ as $z \rightarrow-\infty$, whence, using the variation of constants formula in (7.1):

$$
u(z) \sim \frac{1}{2} D_{1} \chi^{-4} z^{2}, \quad \text { as } z \rightarrow-\infty .
$$

This implies that $l=\infty$, then $D_{1}=-1$ and the second formula in (7.3) follows. This concludes the proof of the theorem. 
Notice that this theorem does not guarantee the uniqueness of the solution of the problem (7.1)-(7.4). Nevertheless, numerical computation strongly suggests that the function $L(\chi)$ is strictly decreasing and there exists a unique value of $\chi, \chi=\chi_{0}$, such that $L\left(\chi_{0}\right)=0$ (see Figure 7.2).

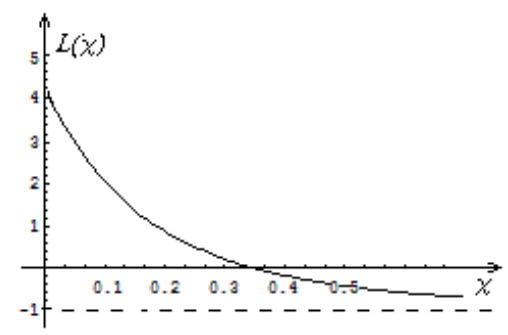

Fig. 7.2: The graph of the function $L(\chi)$ obtained by numerical interpolation using the value of parameters in Table 3.

\section{APPENDIX B: A singular perturbation problem based on the Goldbeter-Segel model}

Using techniques similar to those used in the case of the MG model, we can obtain, for the GS model also, a singular perturbation problem having as underlying kinetics the set of equations (2.6)-(2.8). More precisely, assuming that the extracellular cAMP diffuses, we have the followig system:

$$
\begin{aligned}
& \frac{\partial \alpha}{\partial t}=v-\sigma \phi(\alpha, \gamma) \\
& \frac{\partial \beta}{\partial t}=q \sigma \phi(\alpha, \gamma)-k_{t} \beta \\
& \frac{\partial \gamma}{\partial t}=\frac{k_{t}}{h} \beta-k \gamma+D \frac{\partial^{2} \gamma}{\partial x^{2}}
\end{aligned}
$$

where $D$ is the diffusion coefficient. We introduce the adimensional variables for time and space:

$$
\tau=k_{t} \cdot t, \quad x=\sqrt{\frac{D}{k_{t}}} \cdot \widetilde{x}
$$

and change the variables $\alpha, \beta, \gamma$ with $R, W$ and $U$, in an appropriate manner, in order to obtain a system of equations whose variables are at most of order one. Setting $\xi=\widetilde{x}-\mathcal{V} \tau$, the resulting system is:

$$
\begin{aligned}
-\mathcal{V} R_{\xi}(\xi) & =\kappa-\frac{b R(R+\mu)(U+d)^{2}}{\varepsilon+(R+\mu)^{2}(U+d)^{2}} \\
-\mathcal{V} W_{\xi}(\xi) & =\frac{\Theta R(R+\mu)(U+d)^{2}}{\varepsilon+(R+\mu)^{2}(U+d)^{2}}-W \\
-\mathcal{V} U_{\xi}(\xi) & =\Gamma[W-U]+U_{\xi \xi}
\end{aligned}
$$

The parameters which appear in (8.2)-(8.4) are given in Table 5, that contains also their orders of magnitude and the numerical values (for the numerical values of the parameters which appear in the system (2.6)-(2.8), see $[7])$. 
Table 5. The orders of magnitudes and the numerical values of the parameters of (8.2)-(8.4).

\begin{tabular}{|cccc|}
\hline $\begin{array}{c}\text { Parameters of } \\
(8.2)-(8.4)\end{array}$ & Definition & $\begin{array}{c}\text { Orders of } \\
\text { magnitudes }\end{array}$ & Numerical value \\
\hline$\kappa$ & $\frac{v}{\alpha_{S} \cdot k_{t}}$ & $\ll 1$ & $1.088 \times 10^{-3}$ \\
$b$ & $\frac{\sigma}{\alpha_{S} \cdot k_{t}}$ & $\ll 1$ & $3.265 \times 10^{-2}$ \\
$\mu$ & $\frac{1}{\alpha_{S}}$ & $\ll 1$ & $1.088 \times 10^{-2}$ \\
$d$ & $M^{-1}$ & $\ll 1$ & $3.927 \times 10^{-2}$ \\
$\varepsilon$ & $\frac{L}{\alpha_{S}^{2} M^{2}}$ & $\ll 1$ & 0.187 \\
$\Theta$ & $\frac{q \sigma}{k_{h}} \frac{1}{M}$ & $\simeq 1$ & 1.178 \\
$\Gamma$ & $\frac{k}{k_{t}}$ & $\simeq 1$ & 1 \\
\hline
\end{tabular}

The parameter $\alpha_{S}$ that appear in Table 5 is given by the formula:

$$
\alpha_{S}=\frac{1}{2}\left[\sqrt{\left(\frac{\sigma}{\sigma-v}\right)^{2}+\frac{4 v L}{(\sigma-\nu)\left(\frac{q \nu}{k h}+1\right)^{2}}}-\frac{\sigma-2 \nu}{\sigma-\nu}\right]
$$

and $M$ is the largest root of the equation $\left(1+\alpha_{S}\right)(1+\gamma)^{2}\left[\alpha_{S} q \sigma-k h\left(1+\alpha_{S}\right) \gamma\right]-k h L \gamma=0$.

We obtain that the speed and the width of the pulse, as functions of the initial biochemical parameters, are:

$$
\begin{aligned}
& V_{c A M P \text { pulse }}=\sqrt{D} \cdot\left(\frac{\alpha_{S} q \sigma k_{t}}{h \sqrt{L}}\right)^{\frac{1}{4}} \cdot \chi \\
& x_{\text {width }}=\sqrt{D} \cdot \frac{\alpha_{s}}{\sigma} \cdot\left(\frac{\alpha_{s} q \sigma k_{t}}{h \sqrt{L}}\right)^{\frac{1}{4}} \cdot \chi
\end{aligned}
$$

where $\chi$ is the unique eigenvalue solving the non-linear problem

$$
\begin{aligned}
-w_{z} & =\frac{u^{2}}{1+u^{2}} \quad-\infty<z<\infty \\
-u_{z} & =u_{z z}+\chi^{-4} w \quad-\infty<z<\infty \\
w & \sim-z, \quad u \sim \frac{\chi^{-4}}{2} z^{2}, \quad z \rightarrow-\infty \\
\lim _{z \rightarrow \infty} w & =0, \quad \lim _{z \rightarrow \infty} u=0
\end{aligned}
$$

For the numerical values of the parameters as in Table 5, the corresponding eigenvalue $\chi$ of the problem (8.7)-(8.10), numerically computed, is $\chi \approx 3.76878$. Assuming that the diffusion coefficient for cAMP is $D=$ $4 \times 10^{-6} \mathrm{~cm}^{2} / \mathrm{sec}$, we obtain that $V_{c A M P \text { pulse }} \approx 3685.56 \mu \mathrm{m} / \mathrm{min}$ and $x_{\text {width }} \approx 4703.4 \mu \mathrm{m}$. It is obviously that these numerical values disagrees with the experimental values. The reason is that, as explained in the Introduction, the assumptions in which the GS model is based are not realistic from the biological point of view. Nevertheless, we have preferred to provide a sketch of the analysis above to point out the mathematical analogies existing between both the MG and the GS models.

\section{References}

[1] Bonner, J.T.: The cellular slime molds, Princeton University Press, Princeton, New Jersey, 1967

[2] Dallon J.C., Othmer, H.G.: A discrete cell model with adaptive signalling for aggregation of Dictyostelium discoideum, Phil. Trans. R. Soc. Lond. B 352, 391-417 (1997)

[3] Durston, A.J.: Pacemaker activity during aggregation in Dictyostelium discoideum, Developmental Biology 37, 225-235 (1974)

[4] Durston, A.J.: Pacemaker mutants of Dictyostelium discoideum, Developmental Biology 38, 308-319 (1974)

[5] Falcke, M., Levine, H.: Pattern selection by gene expression in Dictyostelium discoideum, Phys. Rev. Lett. 80, 3875-3878 (1998) 
[6] Gerisch, G., Hülser, D., Malchow, D., Wick, U.: Cell communication by periodic cyclic-AMP pulses, Phil. Trans. R. Soc. Lond. B 272,181-192 (1975)

[7] Goldbeter, A., Segel, L.A.: Unified mechanism for relay and oscillation of cyclic AMP in Dictyostelium discoideum, Proc. Natl. Acad. Sci. USA 74, 1543-1547 (1977)

[8] Grindrod, P.: Patterns and Waves. The theory and applications of reaction-diffusion equations., Clarendon Press, Oxford (1991)

[9] Hagan, P.S., Cohen, M.S.: Diffusion-induced morphogenesis in the development of Dictyostelium, J. Theor. Biol. 93, 881-908 (1981)

[10] Höfer, T., Sherratt, J.A., Maini, P.K.: Cellular pattern formation during Dictyostelium aggregation, Physica D 85, 425-444 (1995)

[11] Keener, J., Sneyd, J.: Mathematical Physiology, Springer-Verlag, New York, (1998)

[12] Lee, K.J., Cox, E.C., Goldstein, R.E.: Competing patterns of signalling activity in Dictyostelium discoideum, Phys. Rev. Lett. 76, 1174-1177 (1996)

[13] Lee, K.J., Wave pattern selection in a excitable system, Phys. Rev. Lett. 79, 2907-2910 (1997)

[14] Levine, H., Tsimring, L., Kessler, D.: Computational modeling of mound development in Dyctiostelium, Physica D 106, 375-388 (1997)

[15] Martiel, J.L., Goldbeter, A.: A model based on receptor desensitization for cyclic AMP signalling in Dictyostelium cells, Biophys. J. 52, 807-828 (1987)

[16] Monk, P.B., Othmer, H.G.: Cyclic AMP oscillations in suspension of Dictyostelium discoideum, Phil. Trans. R. Soc. Lond. 323, 185-224 (1989)

[17] Müller, S.C., Mair, T., Steinbock, O.: Traveling waves in yeast extract and in cultures of Dictyostelium discoideum, Biophysical Chemistry 72, 37-47 (1998)

[18] Murray, J.D.: Mathematical Biology, Second Ed., Springer-Verlag Berlin Heidelberg (1993)

[19] Nanjundiah, V.: Cyclic AMP oscillations in Dictyostelium discoideum: models and observation, Biophysical Chemistry 72, 1-8 (1998)

[20] Othmer, H., Schaap, P.: Oscilatory cAMP signalling in the development of Dictyostelium discoideum, Comments on Theoretical Biology 5, 175-282 (1998)

[21] Rinzel, J., Terman, D.: Propagation phenomena in a bistable reaction-diffusion system, SIAM J. Appl. Math. 42, 1111-1137 (1982)

[22] Othmer, H., Tang, Y.: A G-protein-based model for cAMP dynamics in Dictyostelium discoideum, Math. Biosciences 120, 25-76 (1994)

[23] Tang, Y., Othmer, H.: Excitation, oscillations and wave propagation in a G-protein-based model of signal transduction in Dictyostelium discoideum, Phil. Trans. R. Soc. Lond. B 349, 179-195 (1995)

[24] Winfree, A.T.: Spiral waves of chemical activity, Sci. Amer. 175, 634-636 (1972) 